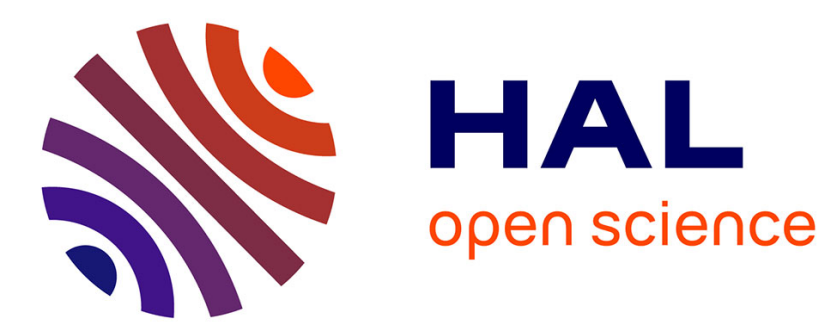

\title{
Universal isomonodromic deformations of meromorphic rank 2 connections on curves
}

Viktoria Heu

\section{To cite this version:}

Viktoria Heu. Universal isomonodromic deformations of meromorphic rank 2 connections on curves. Annales de l'Institut Fourier, 2010, 60 (2), pp.515-549. hal-00377019

\section{HAL Id: hal-00377019 \\ https://hal.science/hal-00377019}

Submitted on 20 Apr 2009

HAL is a multi-disciplinary open access archive for the deposit and dissemination of scientific research documents, whether they are published or not. The documents may come from teaching and research institutions in France or abroad, or from public or private research centers.
L'archive ouverte pluridisciplinaire HAL, est destinée au dépôt et à la diffusion de documents scientifiques de niveau recherche, publiés ou non, émanant des établissements d'enseignement et de recherche français ou étrangers, des laboratoires publics ou privés. 


\title{
Universal isomonodromic deformations of meromorphic rank 2 connections on curves
}

\author{
Viktoria Heu ${ }^{1}$
}

\begin{abstract}
We consider tracefree meromorphic rank 2 connections over compact Riemann surfaces of arbitrary genus. By deforming the curve, the position of the poles and the connection, we construct the global universal isomonodromic deformation of such a connection. Our construction, which is specific to the tracefree rank 2 case, does not need any Stokes analysis for irregular singularities. It is thereby more elementary than the construction in arbitrary rank due to B. Malgrange and I. Krichever and it includes the case of resonant singularities in a natural way.
\end{abstract}

32G34 ( 34M55, 53B05, 32S40, 32G15 )

\section{Introduction}

We consider a meromorphic and tracefree connection $\nabla_{0}$ on a holomorphic rank 2 vector bundle $E_{0}$ over a compact Riemann surface $X_{0}$ of genus $g$. In local trivialization charts for $E_{0}$, the connection $\nabla_{0}$ is defined by $\mathrm{d}-A_{0}$, where $A_{0}$ is a $2 \times 2$-matrix whose entries are meromorphic 1-forms such that $\operatorname{tr}\left(A_{0}\right) \equiv 0$. Such a connection $\left(E_{0}, \nabla_{0}\right)$ will be considered up to holomorphic gauge-transformations of the vector bundle (see section 2.1).

Roughly speaking, an isomonodromic deformation of the initial connection $\left(E_{0} \rightarrow\right.$ $\left.X_{0}, \nabla_{0}\right)$ is an analytic, topologically trivial deformation $\left(E_{t} \rightarrow X_{t}, \nabla_{t}\right)_{t \in T}$ such that the monodromy data are constant. By topologically trivial deformation we mean that the analytic family $\pi: \mathcal{X} \rightarrow T$ of Riemann surfaces with fiber $\pi^{-1}(t)=X_{t}$ has contractible parameter space $T$ and is provided with disjoint sections $\mathcal{D}^{i}: T \rightarrow \mathcal{X}$ for $i \in\{1, \ldots, m\}$, which correspond to the polar locus of the family of connections. With the notations $\mathcal{D}=\sum_{i=1}^{m} \mathcal{D}^{i}$ and $\mathcal{D}=\left(D_{t}\right)_{t \in T}$, the family $\left(X_{t} \backslash D_{t}\right)_{t \in T}\left(\operatorname{resp} .\left(X_{t}, D_{t}\right)_{t \in T}\right)$ can be seen as a family of marked punctured Riemann surfaces (resp. $m$-pointed marked Riemann surfaces).

In the non-singular or logarithmic case (poles of order 1 ), the monodromy data reduce to the monodromy representation $\pi_{1}\left(X_{0} \backslash D_{0}\right) \rightarrow \mathrm{SL}(2, \mathbf{C})$, where $D_{0}$ is the polar locus of the initial connection. In this case, a deformation $\left(E_{t} \rightarrow X_{t}, \nabla_{t}\right)_{t \in T}$ of the curve, the fibre bundle and the connection is called isomonodromic if it is induced by a flat logarithmic connection $(\mathcal{E} \rightarrow \mathcal{X}, \nabla)$. In the general meromorphic case (poles of arbitrary order), one usually adds Stokes matrices to the monodromy data (see papers of B. Malgrange, J. Palmer and I. Krichever), therefore needing a non-resonance condition. In the non-resonant case, a

\footnotetext{
${ }^{1}$ This paper is part of the author's doctoral thesis written at the University of Rennes 1 under the supervision of F. Loray, and was partially supported by ANR SYMPLEXE BLAN06-3-137237
} 
deformation $\left(E_{t} \rightarrow X_{t}, \nabla_{t}\right)_{t \in T}$ is called isomonodromic (and iso-Stokes) if the order of the poles is constant along the deformation, and it is induced by a flat meromorphic connection over $\mathcal{X}$ whose connection matrix $A$ satisfies

$$
(\mathrm{d} A)_{\infty} \leq(A)_{\infty}
$$

where $(\cdot)_{\infty}$ denotes the (effective) polar divisor. If $\left(x_{1}, \ldots, x_{N}\right)$ are local coordinates in which the polar locus is given by $\left\{x_{1}=0\right\}$, then condition (1) means that the connection matrix $A$ takes the form

$$
A=M_{1} \frac{\mathrm{d} x_{1}}{x_{1}^{l}}+\sum_{i=2}^{N} M_{i} \frac{\mathrm{d} x_{i}}{x_{1}^{l-1}},
$$

where $M_{i}$, for $i \in\{1, \ldots, N\}$, is a holomorphic $2 \times 2$-matrix whose entries are holomorphic functions, and $l$ is the order of the pole. It turns out (see section 2.2) that if we consider the $\mathfrak{s l}(2, \mathbf{C})$-case and if the order of the pole does not vary along $\left\{x_{1}=0\right\}$, then condition (1) is equivalent to the existence of local coordinates in which the connection is gauge-equivalent to a constant one:

$$
A=M_{1}\left(x_{1}\right) \frac{\mathrm{d} x_{1}}{x_{1}^{l}}
$$

Our definition of isomonodromic deformations shall be this latter one, which is specific to the $\mathfrak{s l}(2, \mathbf{C})$-case and which also takes sense in the $\mathfrak{s l}(2, \mathbf{C})$-resonant case (see section 2.2).

This paper is devoted to the construction of the global universal isomonodromic deformation $(\mathcal{E} \rightarrow \mathcal{X}, \nabla)$ over $\mathcal{X} \rightarrow T$ of the initial connection, which is carried out in section 3 . It is universal in the sense that any other isomonodromic deformation of the same initial connection can be obtained by inverse image under some classifying application from the universal objet (see the theorem 1 below for a more precise statement). It is global in the sense that this universal property does not hold only for germs of isomonodromic deformations. In the non-singular or logarithmic case (see sections 3.1 and 3.2), the parameter space $T$ of the universal isomonodromic deformation is the Teichmüller space $\mathcal{T}$ associated to the marked curve $X_{0}$ with the set of distinguished points $D_{0}$ which corresponds to the polar set of $\nabla_{0}$. Then $(\mathcal{E}, \nabla)$ is the unique flat logarithmic extension of $\left(E_{0}, \nabla_{0}\right)$ over the universal Teichmüller curve. In the case of irregular poles there are non-trivial isomonodromic deformations of the initial connection, which are fixing the curve and the poles. The parameter space $T$ of the universal isomonodromic deformation in the general case (see section 3.3) is the product of the Teichmüller space $\mathcal{T}$ of the $m$-pointed curve, with spaces of convenient jets of diffeomorphisms at the poles. The dimension of this parameter space $T$ is

$$
\max \{0,3 g-3+n\},
$$

where $n$ is the number of poles counted with multiplicity, except for the special case $(g, n)=$ $(1,0)$, where we have $\operatorname{dim}(T)=1$. The universal property of the universal isomonodromic deformation $(\mathcal{E} \rightarrow \mathcal{X}, \nabla)$ states as follows : 
Theorem 1 (Universal property). Let $(\widetilde{\mathcal{E}} \rightarrow \widetilde{\mathcal{X}}, \widetilde{\nabla})$ be an isomonodromic deformation of $\left(\widetilde{E}_{0} \rightarrow \widetilde{X}_{0}, \widetilde{\nabla}_{0}\right)$ with contractible parameter space $\widetilde{T}$ and initial parameter $\tilde{t}_{0}$. Suppose there is an isomorphism $F_{0}:\left(\widetilde{X}_{0}, \widetilde{D}_{0}\right) \stackrel{\sim}{\rightarrow}\left(X_{0}, D_{0}\right)$ of marked curves and an isomorphism $\Psi_{0}$ : $\left(\widetilde{E}_{0}, \widetilde{\nabla}_{0}\right) \stackrel{\sim}{\rightarrow}\left(E_{0}, \nabla_{0}\right)$ of connections given locally by holomorphic gauge transformations $\psi_{0}$ conjugating $\left(\widetilde{E}_{0}, \widetilde{\nabla}_{0}\right)$ to $F_{0}^{*}\left(E_{0}, \nabla_{0}\right)$ :

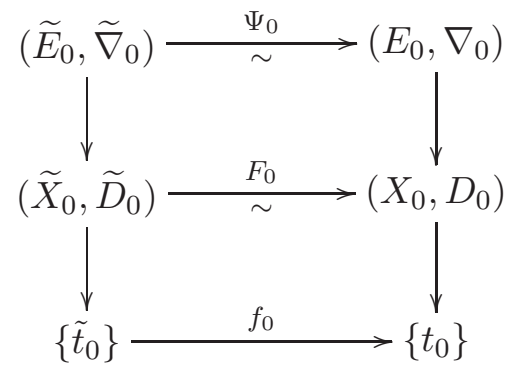

Then there is a triple $(f, F, \Psi)$ extending $\left(f_{0}, F_{0}, \Psi_{0}\right)$ to a commuting diagramm

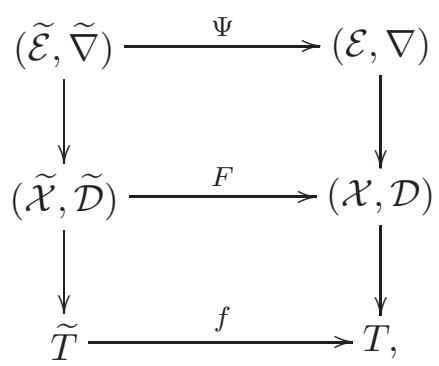

where $(f, F)$ are holomorphic maps such that $\left.F\right|_{\tilde{t}}$ is a biholomorphism of marked Riemann surfaces for each parameter $\tilde{t} \in \widetilde{T}$, and $\Psi$ is given locally by holomorphic gauge transformations $\psi$ conjugating $(\widetilde{\mathcal{E}}, \widetilde{\nabla})$ to $F^{*}(\mathcal{E}, \nabla)$. For each choice of such maps $(f, F)$, there is a unique such isomorphism $\Psi$.

In the non-singular or logarithmic case, the maps $(f, F)$ above will be given by Teichmüller theory. In the general case, the maps $(f, F)$ factorize by the Teichmüller classifying maps. Moreover, the maps $(f, F)$ (and thus the triple $(f, F, \Psi))$ are unique except when there exist non-trivial automorphisms of the marked $m$-pointed base curve, that is in the special cases $(g, m)=(0,0),(0,1),(0,2)$ or $(1,0)$. Otherwise, the uniqueness can be restored by means of a quotient in the construction of the universal isomonodromic deformation by these automorphisms, provided that $(g, n)$ is different from $(0,0),(0,1),(0,2)$ and $(1,0)(c f$. section 4.4). We notice that the remaining cases are undeformable, i.e. $\operatorname{dim}(T)=0$, except for the special case $(g, n)=(1,0)$. The following corollary is an immediate consequence of the construction and the proof of the universal property in section 4 .

Corollary 1. The universal isomonodromic deformation $(\mathcal{E} \rightarrow \mathcal{X}, \nabla)$ of $\left(E_{0} \rightarrow X_{0}, \nabla_{0}\right)$ is also the universal isomonodromic deformation of each of the connections $\left(E_{t} \rightarrow X_{t}, \nabla_{t}\right)$ it contains for a parameter $t \in T$. 
Such a construction of the universal isomonodromic deformation has been done in the non-resonant case for arbitrary rank, using Birkhoff normal form and Stokes matrices, in [Mal83b] (see also [Mal04]), [Mal83c] and [Pal99] for $g=0$, and in [Kri02] for $g \geq 0$. Our construction does not use Stokes analysis, and is in this sense more elementary, but clearly iso-Stokes in the non-resonant case. Based on the observations in [Mal83a] on pages 10-11, B. Malgrange also gave a construction of a germ of a universal isomonodromic deformation for resonant singularities, if the leading term of the connection matrix has only one Jordan block for each eigenvalue (see [Mal86] and [Mal96]). In the $\mathfrak{s l}(2, \mathbf{C})$-case, each resonant singularity is clearly of that type. Our elementary geometric approach unifies all these constructions in the special case of tracefree rank 2 connections.

Another possible approach, that we omit in this work, is the Kodaira-Spencer method. After projectivization of the fibre bundle $\mathcal{E}$, the flat connection $\nabla$ defines a codimension 1 equisingular unfolding on $\mathbf{P}(\mathcal{E})$ in terms of [Mat91], [MN94]. The obstruction space is given by $\mathrm{H}^{1}\left(X_{0}, \Theta_{X_{0}}\left(D_{0}\right)\right)$, where $D_{0}$ is the effective polar divisor of $\nabla_{0}$ and $\Theta$ is the sheaf of holomorphic vector bundles. The dimension of $\Theta_{X_{0}}\left(D_{0}\right)$ is $3 g-3+\operatorname{deg}\left(D_{0}\right)$. The main result of [MN94] insures the existence of a local Kuranishi space. This is a germ of our parameter space.

\section{Definitions and elementary properties}

In the following, we shall always denote by $M$ a complex manifold and by $X$ a compact Riemann surface. We denote by $\mathcal{O}$ the sheaf of holomorphic functions on $M$ (resp. $X$ ) and by $\Omega \otimes \mathcal{M}$ the sheaf of meromorphic 1 -forms on $M$ (resp. $X$ ).

\subsection{Flat meromorphic connections and monodromy}

Let $E$ be a holomorphic rank $r$ vector bundle over $M$. The bundle $E$ is given by a trivialization atlas $\left(U_{i}\right)$ on $M$ with transition maps $\varphi_{i j}$, providing trivialization charts $U_{i} \times \mathbf{C}^{r}$ with local coordinates $\left(z_{i}, Y_{i}\right)$, and transition maps $\left(\Phi_{i j}\right)=\left(\varphi_{i j}, \phi_{i j}\right)$ satisfying

$$
\left(z_{i}, Y_{i}\right)=\left(\varphi_{i j}\left(z_{j}\right), \phi_{i j}\left(z_{j}\right) \cdot Y_{j}\right)
$$

where $\phi_{i j} \in \operatorname{GL}\left(r, \mathcal{O}\left(U_{i} \cap U_{j}\right)\right)$. Later on, we also denote by $E$ the global space of the vector bundle.

A meromorphic connection $\nabla$ on $E$ associates to each trivialization chart $U_{i} \times \mathbf{C}^{r}$ of $E$ with coordinates $\left(z_{i}, Y_{i}\right)$ a system

$$
\mathrm{d} Y_{i}=A_{i}\left(z_{i}\right) \cdot Y_{i}
$$

with $A_{i} \in \mathfrak{g l}\left(2, \Omega \otimes \mathcal{M}\left(U_{i}\right)\right)$, such that the connection matrices $A_{i}$ glue together by means of the transition maps $\left(\Phi_{i j}\right)$ :

$$
A_{i} \circ \varphi_{i j}=\phi_{i j} A_{j} \phi_{i j}^{-1}+\mathrm{d} \phi_{i j} \phi_{i j}^{-1}
$$


Biholomorphic coordinate transformations $\tilde{z}_{i}=\varphi_{i}\left(z_{i}\right)$ in the local coordinates of $M$ are conjugating a connection matrix $A_{i}\left(z_{i}\right)$ to

$$
\widetilde{A}_{i}\left(\tilde{z}_{i}\right)=A_{i} \circ \varphi_{i}^{-1}\left(\tilde{z}_{i}\right)
$$

If the coordinates of $M$ are fixed, we consider connections modulo holomorphic gauge transformations $\widetilde{Y}_{i}=\phi_{i}\left(z_{i}\right) Y_{i}$ with $\phi_{i} \in \mathrm{GL}\left(r, \mathcal{O}\left(U_{i}\right)\right)$ on the local charts, conjugating system (2) to d $\widetilde{Y}_{i}=\widetilde{A}_{i}\left(z_{i}\right) \widetilde{Y}_{i}$ with

$$
\widetilde{A}_{i}=\phi_{i} A_{i} \phi_{i}^{-1}+\mathrm{d} \phi_{i} \phi_{i}^{-1}
$$

Otherwise we consider connections modulo holomorphic gauge-coordinate transformations, i.e. combinations of coordinate and gauge transformations. Two connections over the same manifold are called isomorphic, if there is an isomorphism of vector bundles conjugating the associated connection matrices.

The poles of the matrices $A_{i}$ are the poles of the connection $\nabla$. They do not depend on the chart, and the polar divisor $(\nabla)_{\infty}$ is well defined. We shall denote by $D$ the reduced polar divisor. The connection $\nabla$ is said to be a non-singular (resp. logarithmic) connection if it has no poles (resp. if it has only simple poles and its connection matrices $A_{i}$ satisfy $\left.\left(\mathrm{d} A_{i}\right)_{\infty} \leq\left(A_{i}\right)_{\infty} \cdot\right)$

A connection is flat or integrable, if the connection matrices $A_{i}$ satisfy $\mathrm{d} A_{i} \equiv A_{i} \wedge A_{i}$. Equivalently, the connection is flat if each non-singular point has a small neighborhood such that there is a gauge transformation $(z, \tilde{Y})=(z, \phi(z) \cdot Y)$ conjugating the connection matrix to the trivial connection matrix $\widetilde{A}_{i}=0$.

We can choose a fundamental solution $\mathrm{S}$, that is a basis of the space of local solutions in some base point in the set of non-singular points $M^{*}=M \backslash D$. Then analytic continuation along a closed path $\gamma$ in $M^{*}$ provides another fundamental solution $S^{\prime}=\rho^{-1}(\gamma) S$, where $\rho(\gamma)$ is called the monodromy along the path $\gamma$. In that way we get a monodromy representation $\rho: \pi_{1}\left(M^{*}\right) \rightarrow \mathrm{GL}(r, \mathbf{C})$ which will be considered modulo conjugacy of the image of $\rho$ by an element of $\operatorname{GL}(r, \mathbf{C})$.

Each meromorphic rank $r$ connection $\nabla$ on $E$ induces a trace connection $\operatorname{tr}(\nabla)$ on the line bundle $\operatorname{det}(E)$, given by

$$
\mathrm{d} y_{i}=\operatorname{tr}\left(A_{i}\left(z_{i}\right)\right) \cdot y_{i}
$$

We say a connection is tracefree, if its trace connection is the trivial connection $\mathrm{d} y \equiv 0$ on the trivial line bundle $M \times \mathbf{C}$. For tracefree connections, it is possible to choose transition maps with $\phi_{i j} \in \operatorname{SL}\left(r, \mathcal{O}\left(U_{i} \cap U_{j}\right)\right)$. For tracefree connections we thus will only consider gauge transformations $\phi_{i}$ in $\operatorname{SL}\left(r, \mathcal{O}\left(U_{i}\right)\right)$.

\subsection{Isomonodromic deformations}

Let $\left(X_{t}\right)_{t \in T}$ be an analytic family of marked Riemann surfaces (cf. [Nag88], page 347) which is given by a submersion $\pi: \mathcal{X} \rightarrow T$ with contractible parameter space $T$. Let 
$(\mathcal{E}, \nabla)$ be a meromorphic connection (not necessarily flat) on $\mathcal{X}$, inducing an analytic family $\left(E_{t} \rightarrow X_{t}, \nabla_{t}\right)_{t \in T}$. For each parameter $t \in T$ denote by $D_{t}$ the polar set of the connection $\left(E_{t} \rightarrow X_{t}, \nabla_{t}\right)$. We only consider the case where $\mathcal{D}=\left(D_{t}\right)_{t \in T}$ is a smooth divisor on $\mathcal{X}$, which is transversal to the parameter $t$. In particular, $\mathcal{X} \backslash \mathcal{D} \rightarrow T$ then is a family of marked punctured Riemann surfaces and for each parameter $t \in T$ the inclusion map $X_{t} \backslash D_{t} \longleftrightarrow \mathcal{X} \backslash \mathcal{D}$ defines an isomorphism $\pi_{1}\left(X_{t} \backslash D_{t}\right) \cong \pi_{1}(\mathcal{X} \backslash \mathcal{D})$. We call $\left(E_{t} \rightarrow X_{t}, \nabla_{t}\right)_{t \in T}$ a topologically trivial, analytic family of connections.

A topologically trivial, analytic family $\left(E_{t} \rightarrow X_{t}, \nabla_{t}\right)_{t \in T}$ is called an isomonodromic family, if it is induced by a flat connection over the total space $\mathcal{X} \rightarrow T$ of the family of curves. Along an isomonodromic family, the monodromy representation is constant. An isomonodromic deformation is a special case of isomonodromic families, which is induced by some initial connection $\left(E_{0} \rightarrow X_{0}, \nabla_{0}\right)$, and where the Stokes-data are also constant along the deformation. Yet Stokes data are well-defined only in the non-resonant case, i.e. if the leading term of the connection matrix of the initial connection has only distinct eigenvalues. Usually (c.f. [Mal83b], [Mal83c], [Pal99], [Kri02]), isomonodromic deformations of tracefree rank 2 connections are defined as follows.

Definition 1. Let $\left(E_{0} \rightarrow X_{0}, \nabla_{0}\right)$ be a non-resonant, tracefree rank 2 connection on a Riemann surface $X_{0}$. A topologically trivial, analytic deformation $\left(E_{t} \rightarrow X_{t}, \nabla_{t}\right)_{t \in T}$ of this initial connection is called an isomonodromic deformation, if

- for each parameter $t$, the order of the poles of $\nabla_{t}$ is equal to the order of the poles of $\nabla_{0}$ and

- $\left(E_{t} \rightarrow X_{t}, \nabla_{t}\right)_{t \in T}$ is induced by a flat connection $(\mathcal{E} \rightarrow \mathcal{X}, \nabla)$, whose connection matrix A satisfies the following transversality-condition :

$$
(\mathrm{d} A)_{\infty} \leq(A)_{\infty}
$$

Remark 1. Consider a flat tracefree connection of rank 2 on a smooth family of vector bundles over Riemann surfaces with smooth polar divisor $\mathcal{D}$ (as a set). Then any irreducible component $\mathcal{D}^{i}$ of the polar divisor $\mathcal{D}$ not satisfying the transversality condition (3) is projectively apparent in the following sense : after a bimeromorphic transformation, the polar divisor of the associated projective connection becomes $\mathcal{D} \backslash \mathcal{D}^{i}$ (as a set) (see [LP07], page 736).

Let $(\mathcal{E} \rightarrow \mathcal{X}, \nabla)$ be a flat connection inducing an isomonodromic deformation $\left(E_{t} \rightarrow\right.$ $\left.X_{t}, \nabla_{t}\right)_{t \in T}$ of an initial $\mathfrak{s l}(2, \mathbf{C})$-connection $\left(E_{0} \rightarrow X_{0}, \nabla_{0}\right)$. Locally over each non-singular point of $\mathcal{X}$, the connection $\nabla$ is given by systems $\mathrm{d} Y \equiv 0$. In particular, any local solution in a non-singular point is automatically transverse to the parameter $t \in T$, i.e. transverse to $\{t=$ const $\}$. We shall see that the transversality condition (3), together with the constancy of the order of the poles and our initial hypothesis of the divisor $\mathcal{D}$ being transverse to 
the parameter implies local constancy, which is a certain local product structure of the connection with the parameter space even at the singular points. Let us now precise the notion of local constancy.

On smooth families $\left(X_{t}\right)_{t \in T}$ of marked Riemann surfaces we shall always denote by $(t, x) \in W \times U$ local trivialization coordinates, with $t_{1} \in W \subset T$ and $x \in U \subset X_{t_{1}}$. By our initial hypothesis, the distinguished points $\mathcal{D}=\left(D_{t}\right)_{t \in T}$ can locally be given by $\{x=0\}$ in such coordinates. By gauge-coordinate transformations in such coordinates $(t, x, Y) \in W \times U \times \mathbf{C}^{2}$ with $W \subset T$, we will always mean gauge-coordinate transformations fixing the parameter $t$ :

$$
(\tilde{t}, \tilde{x}, \tilde{Y})=(t, \varphi(t, x), \phi(t, x) \cdot Y) .
$$

Definition 2. A flat connection $\nabla$ on a smooth family of holomorphic vector bundles $\left(E_{t} \rightarrow X_{t}\right)_{t \in T}$ is called locally constant if locally in each point of the total space $\mathcal{X}$ of the curve deformation, the connection matrix does not depend on the parameter $t \in T$, up to a convenient gauge-coordinate transformation.

Remark 2. In other words, on open sets as above, there are submersions $\varphi: W \times U \rightarrow U$ transversal to the parameter, such that $\nabla$ is gauge-equivalent to the pull-back $\varphi^{*}\left(\left.\nabla\right|_{t=t_{1}}\right)$.

This means that up to an appropriate gauge-coordinate transformation on $W \times U \times \mathbf{C}^{2}$, the system $\mathrm{d} Y=A(x) Y \mathrm{~d} x$ defining $\left.\nabla\right|_{t=t_{1}}$ over $U$ defines $\nabla$ over $W \times U$ as well. In this sense, $\nabla$ can locally be seen as the product of an initial connection with the parameter space.

Proposition 1. Let $(\mathcal{E} \rightarrow \mathcal{X}, \nabla)$ be a flat tracefree rank 2 connection on $(\mathcal{X}, \mathcal{D}) \rightarrow T$, such that the leading term of the connection matrix $A$ has no zeros along the polar divisor. Then the connection $(\mathcal{E} \rightarrow \mathcal{X}, \nabla)$ is locally constant if, and only if, its connection matrix satisfies the transversality condition (3).

Remark 3. The transversality condition is strictly weaker to the condition of local constancy, if we consider connections of rank greater than 2 or rank 2 connections with nontrivial trace.

In order to prove proposition 1, we will use the following lemma.

Lemma 1. Let $(\mathcal{E} \rightarrow \mathcal{X}, \nabla)$ be a flat tracefree rank 2 connection on $(\mathcal{X}, \mathcal{D}) \rightarrow T$ which satisfies the transversality condition (3) and whose order of the poles is constant. Then for each point $t_{1}$ of $T$ there is a neighborhood $W$ of $t_{1}$ and for each point of the polar divisor in the fibre over $t_{1}$, there is a neighborhood $W \times U \times \mathbf{C}^{2}$ with coordinates $(t, x, Y)$ where the connection $\nabla$ is given by systems of normal form

$$
\mathrm{d} Y=\frac{1}{x^{l}}\left(\begin{array}{cc}
0 & 1 \\
c(x) & 0
\end{array}\right) Y \mathrm{~d} x
$$

not depending on $t$, where $c$ is a holomorphic function on $U$. 
Proof: On a local chart $W \times U \times \mathbf{C}^{2}$, let $\nabla$ be given by a system

$$
\mathrm{d} Y=A(t, x) Y \quad \text { with } \quad A=\left(\begin{array}{cc}
a & b \\
c & -a
\end{array}\right)
$$

If $\nabla_{t}$ has a pole of order $l$ at $\{x=0\}$ for each parameter $t$ in a neighborhood of $t_{1}$, then at least one of the 1 -forms $a, b$ or $c$ has a pole of order $l$ at $\{x=0\}$, which remains a pole of order $l$ in restriction to $t_{1}$. We may suppose this is the case for $b$, otherwise we may apply a gauge transformation such as $\widetilde{Y}=\left(\begin{array}{cc}0 & 1 \\ -1 & 0\end{array}\right) Y$ or $\widetilde{Y}=\left(\begin{array}{ll}1 & 1 \\ 0 & 1\end{array}\right) Y$. If the transversality condition is satisfied, then $b$ is of the form $b=\frac{1}{x^{l}}\left(b_{0}(t, x) \mathrm{d} x+x b_{1}(t, x) \mathrm{d} t\right)$, where $b_{0}$ and $b_{1}$ are holomorphic functions with $b_{0}(t, 0) \not \equiv 0$. By our assumption, $b_{0}(t, 0)$ is even nonzero for each parameter $t$ in a small neighborhood of $t_{1}$. Thus $x^{l} b$ defines a non-singular (integrable) foliation transverse to the parameter $t$, and there is a coordinate transformation fixing $\{x=0\}$ and straightening the foliation given by $x^{l} b=0$ to $\mathrm{d} x=0$.

Remark 4. With the notions of remark 2, the submersion $\varphi(t, x)$ defining this coordinate change is given by a first integral of the foliation $x^{l} b=0$.

In other words, up to a coordinate transformation, we make sure that $b$ is of the form $b=$ $\frac{1}{x^{l}} b_{0}(t, x) \mathrm{d} x$, where $b_{0}(t, x)$ has no zeros in a sufficiently small neighborhood of $\left(0, t_{1}\right)$. By a gauge transformation of the form $\widetilde{Y}=\left(\begin{array}{cc}\left(\sqrt{b_{0}}\right)^{-1} & 0 \\ 0 & \sqrt{b_{0}}\end{array}\right) Y$ we obtain $b=\frac{1}{x^{l}} \mathrm{~d} x$. The integrability condition $\mathrm{d} A=A \wedge A$ is equivalent to

$$
\begin{aligned}
& \mathrm{d} a=b \wedge c \\
& \mathrm{~d} b=2 a \wedge b \\
& \mathrm{~d} c=2 c \wedge a .
\end{aligned}
$$

From the special form of $b$ then follows that $a$ is of the form $\frac{a_{0}(t, x)}{x^{l}} \mathrm{~d} x$. By the gauge transformation $\widetilde{Y}=\left(\begin{array}{cc}1 & 0 \\ a_{0} & 1\end{array}\right) Y$, which keeps $b$ invariant, we get even $a \equiv 0$. Then by $(5)$ we have $0=b \wedge c$. Thus $c$ has the form $\frac{c_{0}(t, x)}{x^{l}} \mathrm{~d} x$. Again by (5) we get $\mathrm{d} c=0$. Therefore $c$ does not either depend on $t$ : we have $c=\frac{c_{0}(x)}{x^{l}}$.

Example 1. The condition in the upper lemma that the order of the poles has to be constant is necessary. Consider for example the connection

$$
\mathrm{d} Y=\left(\begin{array}{cc}
-\frac{t}{x^{2}} \mathrm{~d} x+\frac{1}{x} \mathrm{~d} t & 0 \\
0 & \frac{t}{x^{2}} \mathrm{~d} x-\frac{1}{x} \mathrm{~d} t
\end{array}\right) Y
$$

on the trivial bundle over $(\mathbf{C}, 0) \times(\mathbf{C}, 0)$ with coordinates $(t, x)$. It satisfies the transversality condition, but it can not be locally constant since the order of the poles changes for $t=0$. 
Proof of proposition 1: Note first that (3) is satisfied if, and only if, it is satisfied after a gauge-coordinate transformation. Clearly, systems of normal form (4) are locally constant. Conversely, if $\nabla$ is locally constant, the local charts can be chosen in a way that the connection matrices $A$ do not depend on the parameter $t$ :

$$
\mathrm{d} Y=A(x) Y .
$$

Then $\mathrm{d} A$ is zero and has no polar divisor.

Remark 5. Let $(E \rightarrow X, \nabla)$ be a meromorphic, tracefree rank 2 connection over a Riemann surface. The upper proof shows in particular that up to an appropriate holomorphic gauge-transformation this connection is given locally by systems of normal form (4). Then the rational number $\max \left\{l+1-\frac{\nu}{2}, 0\right\}$, where $\nu$ is the greatest integer such that $\frac{c}{x^{\nu}}$ is still holomorphic, is equal to the Katz-rank of the singularity (see [Var96] for a definition of the Katz-rank). The Katz-rank is invariant under meromorphic gauge transformation. Moreover, we see that the Katz-rank is constant along isomonodromic deformations.

According to proposition 1, isomonodromic deformations of tracefree rank 2 connections may be defined alternatively as follows :

Definition 3. A topologically trivial, analytic deformation $\left(E_{t} \rightarrow X_{t}, \nabla_{t}\right)_{t \in T}$ of some initial tracefree rank 2 connection $\left(E_{0} \rightarrow X_{0}, \nabla_{0}\right)$ is called an isomonodromic deformation, if it is induced by a flat, locally constant connection $(\mathcal{E} \rightarrow \mathcal{X}, \nabla)$.

In this paper, we shall use this latter definition of isomonodromic deformations, which is specific to the $\mathfrak{s l}(2, \mathbf{C})$-case. As we shall see, this definition is naturally valid also in the $\mathfrak{s l}(2, \mathbf{C})$-resonant case.

Two isomonodromic deformations of a common initial connection will be called isomorphic, if the associated flat connections are isomorphic. More precisely, two isomonodromic deformations $(\mathcal{E} \rightarrow \mathcal{X}, \nabla)$ and $(\widetilde{\mathcal{E}} \rightarrow \widetilde{\mathcal{X}}, \widetilde{\nabla})$ with polar sets $\mathcal{D}$ and $\widetilde{\mathcal{D}}$ respectively and parameter spaces $T$ and $\widetilde{T}$ respectively are isomorphic, if there is a biholomorphism $f$, an isomorphism $F$ of marked $m$-pointed curves and an isomorphism $\Psi$ of vector bundles extending the isomorphism of the initial connections, such that the following diagramm commutes

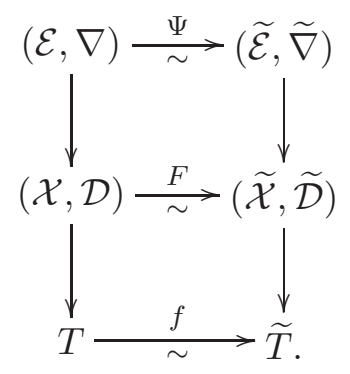


Here $\Psi$ consists locally of gauge-coordinate transformations compatible with $F$ which are conjugating $\nabla$ to $\widetilde{\nabla}$.

It is clear that an isomonodromic deformation $\left(E_{t} \rightarrow X_{t}, \nabla_{t}\right)_{t \in T}$ of some initial connection $\left(E_{0} \rightarrow X_{0}, \nabla_{0}\right)$ can also be seen as an isomonodromic deformation of each of the connections $\left(E_{t} \rightarrow X_{t}, \nabla_{t}\right)$ it contains for a parameter $t \in T$.

Remark 6. We would like to stress that the gauge transformations we defined for these flat families of connections are not only holomorphic families of gauge transformations, but global holomorphic gauge transformations of the flat connection on the global bundle $\mathcal{E}$ of the family.

\section{Construction}

Let $X_{0}$ be a Riemann surface of genus $g$. Let $\nabla_{0}$ be a meromorphic tracefree rank 2 connection on $E_{0} \rightarrow X_{0}$ with $m$ poles of multiplicity respectively $n_{1}, \ldots n_{m}$, given in local coordinates $x^{i}$ by $\left\{x^{i}=0\right\}$. Denote by $n=n_{1}+\ldots+n_{m}$ the number of poles counted with multiplicity and denote by $D_{0}=\sum_{i=1}^{n}\left\{x^{i}=0\right\}$ the polar set in $X_{0}$ of this connection. By $X_{0}^{*}$ we denote the set of non-singular points $X_{0} \backslash D_{0}$. Let $\rho: \pi_{1}\left(X_{0}^{*}\right) \rightarrow \operatorname{SL}(2, \mathbf{C})$ be the monodromy representation of $\nabla_{0}$. In this section, we construct the universal isomonodromic deformation $(\mathcal{E} \rightarrow \mathcal{X}, \nabla)$ of $\left(E_{0} \rightarrow X_{0}, \nabla_{0}\right)$ over a base curve $\mathcal{X} \rightarrow T$. Consider the universal curve of marked $m$-pointed Riemann surfaces $\mathcal{X}_{\mathcal{T}} \rightarrow \mathcal{T}$, parametrized by $\left(\mathcal{T}, \tau_{0}\right)$ being the Teichmüller space Teich $(g, m)$ with initial parameter $\tau_{0}$ corresponding to $X_{0}$ with the distinguished set $D_{0}$, as in [Nag88], page 322 .

Remark 7. The dimension of the Teichmüller space Teich $(g, m)$ is

$$
\begin{array}{cl}
3 g-3+m & \text { if } g \geq 2 \\
\max \{m, 1\} & \text { if } g=1 \\
\max \{m-3,0\} & \text { if } g=0
\end{array}
$$

We shall denote by $\mathcal{D}_{\mathcal{T}}=\sum_{i=1}^{m} \mathcal{D}_{\mathcal{T}}^{i}$ the submanifold of $\mathcal{X}_{\mathcal{T}}$ corresponding to the distinguished points and their deformations. Consider the exact sequence of homotopy groups associated to the fibration $\mathcal{X}_{\mathcal{T}}^{*} \rightarrow \mathcal{T}$, where $\mathcal{X}_{\mathcal{T}}^{*}=\mathcal{X}_{\mathcal{T}} \backslash \mathcal{D}_{\mathcal{T}}$. Since the Teichmüller space $\mathcal{T}$ is contractible ( $c f$. [Hub06], page 274), the natural inclusion map of $X_{0}^{*}$ into $\mathcal{X}_{\mathcal{T}}^{*}$ induces an isomorphism between their fundamental groups. In that way, we can consider $\rho$ as a representation of $\pi_{1}\left(\mathcal{X}_{\mathcal{T}}^{*}\right)$ as well.

If the initial connection $\left(E_{0} \rightarrow X_{0}, \nabla_{0}\right)$ has only logarithmic poles in $D_{0}$, we shall see that it extends in a unique way to an integrable logarithmic connection on $\mathcal{X}_{\mathcal{T}}$ with poles in $\mathcal{D}_{\mathcal{T}}$. This will define the universal isomonodromic deformation in the logarithmic case. In the case of a non-logarithmic initial connection, there still is an integrable locally constant connection on $\mathcal{X}_{\mathcal{T}}$ with poles in $\mathcal{D}_{\mathcal{T}}$ extending $\left(E_{0} \rightarrow X_{0}, \nabla_{0}\right)$, but this connection will no 
longer be unique. Indeed, each pole of order $l$ will contribute $l-1$ degrees of freedom in the construction. We thereby get a universal isomonodromic deformation of dimension

$$
3 g-3+m+(n-m)=3 g-3+n
$$

if $(g, m) \neq(0,0),(0,1),(0,2),(1,0)$. The universal isomonodromic deformation will be global, due to the existence of tubular neighborhoods $\mathcal{U}_{\mathcal{T}}^{i}$ of $\mathcal{D}_{\mathcal{T}}^{i}$ in $\mathcal{X}_{\mathcal{T}}$.

\subsection{Non-singular case (arbitrary rank)}

In the non-singular case, the existence of the universal isomonodromic deformation follows immediately from the classical Riemann-Hilbert correspondence recalled by P. Deligne in [Del70].

Theorem 2 (Riemann-Hilbert correspondence). Let $M \ni z_{0}$ be a complex manifold. The functor of monodromy with respect to the fibre over $z_{0}$ defines an equivalence between the category of non-singular integrable rank $r$ connections on $M$ and the category of representations from $\pi_{1}\left(M, z_{0}\right)$ to $\mathrm{GL}(F)$, where $F$ is an $r$-dimensional $\mathbf{C}$-vector space. At the left side, the homomorphisms we consider are isomorphisms; at the right side, we consider isomorphisms between the vector spaces which identify two representations.

Proof: Let us prove the essential surjectivity first. For a given representation $\rho: \pi_{1}\left(M, z_{0}\right) \rightarrow$ $\mathrm{GL}(F)$, we can construct an associated connection over $M$ by suspension. We choose an isomorphism $F \cong \mathbf{C}^{r}$. The induced representation $\pi_{1}\left(M, z_{0}\right) \rightarrow \operatorname{GL}\left(\mathbf{C}^{r}\right)$ shall also be denoted by $\rho$. Let $\widetilde{M}$ be the universal cover of the complex manifold $M$. Let $\widetilde{\nabla}$ be the trivial connection $\mathrm{d} \widetilde{Y} \equiv 0$ on the trivial bundle $\widetilde{M} \times \mathbf{C}^{r}$ with coordinates $(\tilde{z}, \widetilde{Y})$ over $\widetilde{M}$. Now the fundamental group $\pi_{1}(M)$ is naturally acting on $\widetilde{M}$. We may further define an action on $\widetilde{M} \times \mathbf{C}^{r}$ in the following way :

$$
\gamma \cdot(\tilde{z}, \tilde{Y})=(\gamma \cdot \tilde{z}, \rho(\gamma) \cdot \tilde{Y})
$$

Since the monodromy matrices $\rho(\gamma)$ are constant, the connection $\mathrm{d} \widetilde{Y}=0$ can naturally be pushed down to the quotient of this action. Thereby we define a non-singular connection on an implicitely defined vector bundle $E$ over $M$. This connection has monodromy $\rho$ with respect to the fibre over $z_{0}$.

In order to prove the full faithfulness of the monodromy functor, let $(E, \nabla)$ and $(\widetilde{E}, \widetilde{\nabla})$ be two non-singular integrable rank $r$ connections over $M$ with fibres $F$ and $\widetilde{F}$ respectively over $z_{0}$. We suppose that there is an isomorphism $\psi_{0}: F \stackrel{\sim}{\rightarrow} \widetilde{F}$ which induces an isomorphism of their monodromy representations $\rho$ and $\widetilde{\rho}$. We have to show that there is a unique isomorphism $\psi:(E, \nabla) \stackrel{\sim}{\rightarrow}(\widetilde{E}, \widetilde{\nabla})$ wich induces $\psi_{0}$ in restriction to the fibre over $z_{0}$. We may choose a common atlas of $M$ for both connections. Let $U$ be a small neighborhood of $z_{0} \in M$. Then up to gauge transformations $\phi(z) \cdot Y$ and $\widetilde{\phi}(z) \cdot \widetilde{Y}$ respectively, the connections $\nabla$ and $\widetilde{\nabla}$ are defined in the trivialization charts over $U$ by $\mathrm{d} Y=0$ and $\mathrm{d} \widetilde{Y}=0$ 
respectively. Horizontal sections are constant in these coordinates, and thus $\psi_{0}$ induces a unique isomorphism $\psi=\widetilde{\phi}^{-1} \circ \psi_{0} \circ \phi$ between the connections in restriction to $U$. This isomorphism can be continued analytically and since the analytic continuations of $\psi_{0} \circ \phi$ and $\widetilde{\phi}$ give rise to the same monodromy representation $\widetilde{\rho}$, the analytic continuation of $\psi$ has trivial monodromy. Thus $\psi_{0}$ defines a unique isomorphism $\psi$ over $M$.

Under the notations of the beginning of this section, recall that the natural inclusion map $X_{0} \longrightarrow \mathcal{X}_{\mathcal{T}}$ defines an isomorphism of the fundamental classes $\pi_{1}\left(X_{0}, x_{0}\right) \cong$ $\pi_{1}\left(\mathcal{X}_{\mathcal{T}}, x_{0}\right)$. We thus get the following corollary from the classical Riemann-Hilbert correspondence.

Corollary 2. If $\left(E_{0}, \nabla_{0}\right)$ is non-singular over $X_{0}$, then this connection extends to a flat non-singular connection $(\mathcal{E}, \nabla)$ over the universal Teichmüller curve $\mathcal{X}_{\mathcal{T}} \rightarrow \mathcal{T}$, provided with an isomorphism

$$
\left.\left(\mathcal{E} \rightarrow \mathcal{X}_{\mathcal{T}}, \nabla\right)\right|_{\tau=\tau_{0}} \cong\left(E_{0} \rightarrow X_{0}, \nabla_{0}\right) .
$$

This object is unique, up to unique isomorphism, i.e. given another flat connection over $\mathcal{X}_{\mathcal{T}}$ together with an isomorphism of $\left(E_{0}, \nabla_{0}\right)$ to its restriction to the initial parameter, the latter isomorphism extends in a unique way to an isomorphism of the connections over $\mathcal{X}_{\mathcal{T}}$.

In the non-singular case, the flat connection $\left(\mathcal{E} \rightarrow \mathcal{X}_{\mathcal{T}}, \nabla\right)$ of the above corollary defines the universal isomonodromic deformation of $\left(E_{0} \rightarrow X_{0}, \nabla_{0}\right)$. Remark that for each parameter $\tau \in \mathcal{T}$, this universal object induces the unique (modulo isomorphism) non-singular rank 2 connection $\left(E_{\tau} \rightarrow X_{\tau}, \nabla_{\tau}\right)$ over $X_{\tau}$, having monodromy $\rho$ (modulo conjugacy).

\subsection{Logarithmic case (arbitrary rank)}

There is also a Riemann-Hilbert correspondence for flat logarithmic connections on a complex manifold $M$ with a given normal crossing divisor $D$, if we fix the residus in each pole, i.e. the eigenvalues of the polar part of the connection matrix. If there is no resonance, i.e. if the residues in each pole do not differ in non-zero integers, then the monodromy functor establishes an equivalence between the category of flat logarithmic rank $r$ connections over $M$ with polar divisor $D$ and the given residues (modulo holomorphic gauge transformations) and the category of representations of $\pi_{1}(M \backslash \mathcal{D})$ in $\mathrm{GL}(F)$, where $F$ is an $r$-dimensional $\mathbf{C}$-vector space (see [Del70], [Kat76], [Bri04]). In the rank 2 case, it is even possible to avoid the non-resonance condition if one considers some more precise monodromy data (indicating the position of special lines). Together with the observation that the residues and the special lines can not change along an isomonodromic deformation, this Riemann-Hilbert correspondence immediately provides the universal isomonodromic deformation as it did in the non-singular case. Yet we prefer to follow the more elegant construction B. Malgrange gave in his article [Mal86] in order to prove the following theorem, which remains valid even in arbitrary rank (the notations are those of the beginning of the section). 
Theorem 3. If $\left(E_{0}, \nabla_{0}\right)$ is logarithmic over $X_{0}$ with simple poles in $D_{0}$, then this connection extends to a flat logarithmic connection $(\mathcal{E}, \nabla)$ with polar set $\mathcal{D}_{\mathcal{T}}$ over the universal Teichmüller curve $\mathcal{X}_{\mathcal{T}} \rightarrow \mathcal{T}$, provided with an isomorphism

$$
\left.\left(\mathcal{E} \rightarrow \mathcal{X}_{\mathcal{T}}, \nabla\right)\right|_{\tau=\tau_{0}} \cong\left(E_{0} \rightarrow X_{0}, \nabla_{0}\right) .
$$

This object is unique, up to unique isomorphism and shall be called the universal isomonodromic deformation of $\left(E_{0} \rightarrow X_{0}, \nabla_{0}\right)$.

Proof: Let us start with the construction of $\left(\mathcal{E} \rightarrow \mathcal{X}_{\mathcal{T}}, \nabla\right)$. As usual, we denote by $\rho$ the monodromy representation of the initial connection $\left(E_{0}, \nabla_{0}\right)$. Since we can identify $\pi_{1}\left(X_{0}^{*}\right) \cong \pi_{1}\left(\mathcal{X}_{\mathcal{T}}^{*}\right)$, the classical Riemann-Hilbert correpondence provides a non-singular integrable tracefree rank 2 connection $\left(\mathcal{E}^{*} \rightarrow \mathcal{X}_{\mathcal{T}}^{*}, \nabla^{*}\right)$ over the punctured curve $\mathcal{X}_{\mathcal{T}}^{*}=$ $\mathcal{X}_{\mathcal{T}} \backslash \mathcal{D}_{\mathcal{T}}$ having monodromy $\rho$. Recall that this connection extends $\left.\left(E_{0}, \nabla_{0}\right)\right|_{X_{0}^{*}}$ and it is unique up to unique isomorphism.

From the Bers construction of the universal Teichmüller curve (see [Hub06]) follows the existence of tubular neighborhoods $\mathcal{U}_{\mathcal{T}}^{i}$ of $\mathcal{D}_{\mathcal{T}}^{i}$. In other words, there are global coordinates of $\mathcal{D}_{\mathcal{T}}^{i}$ in $\mathcal{U}_{\mathcal{T}}^{i}$, i.e. holomorphic functions $\xi_{i}: \mathcal{U}_{\mathcal{T}}^{i} \rightarrow \mathbf{C}$ such that $\operatorname{div}\left(\xi_{i}\right)=\mathcal{D}_{\mathcal{T}}^{i}$. We may suppose that the function induced by $\xi_{i}$ on $\mathcal{U}_{\mathcal{T}}^{i} \cap X_{0}$ is the coordinate function $x_{i}$ on $U_{0}^{i}=\mathcal{U}_{\mathcal{T}}^{i} \cap X_{0}$. In order to complete the connection $\left(\mathcal{E}^{*}, \nabla^{*}\right)$ at the polar set, we define an integrable connection $\nabla^{i}$ on the trivial vector bundle $\mathcal{E}^{i}$ over the germification of such a tubular neighborhood $\mathcal{U}_{\mathcal{T}}^{i}$ by $\nabla^{i}=\left.\xi_{i}^{*} \nabla\right|_{U_{0}^{i} \backslash D_{0}^{i}}$. In other words, if $\nabla_{0}$ is defined by a system $\mathrm{dY}=A\left(x_{i}\right) Y \mathrm{~d} x_{i}$ over $U_{0}^{i}$, which has a pole in $\left\{x_{i}=0\right\}$, then $\nabla^{i}$ shall be given over $\mathcal{U}_{\mathcal{T}}^{i}$ by the product connection $\mathrm{dY}=A\left(\xi_{i}\right) Y \mathrm{~d} \xi_{i}$, having a pole in $\mathcal{D}_{\mathcal{T}}^{i}$. In restriction to the punctured chart $\mathcal{U}_{\mathcal{T}}^{i *}=\mathcal{U}_{\mathcal{T}}^{i} \backslash \mathcal{D}^{i}$ both $\left(\mathcal{E}^{i}, \nabla^{i}\right)$ and $\left(\mathcal{E}^{*}, \nabla^{*}\right)$ have local monodromy $\rho$. According to the classical Riemann-Hilbert correspondence, there is an isomorphism gluing them into a connection $(\mathcal{E}, \nabla)$ over $\mathcal{X}_{\mathcal{T}}$ such that $\left.\left(\mathcal{E} \rightarrow \mathcal{X}_{\mathcal{T}}, \nabla\right)\right|_{\tau=\tau_{0}}=\left(E_{0} \rightarrow X_{0}, \nabla_{0}\right)$.

We now have to show the uniqueness of this construction. Let $\left(\mathcal{E}^{\prime}, \nabla^{\prime}\right)$ be another flat logarithmic connection extending $\left(E_{0}, \nabla_{0}\right)$ over $\mathcal{X}_{\mathcal{T}}$. Consider the rank 4 vector bundle $\mathcal{E}^{\prime \prime}$ over $\mathcal{X}_{\mathcal{T}}$ defined by $\operatorname{Hom}\left(\mathcal{E}^{\prime}, \mathcal{E}\right)$. Since $\nabla^{\prime}$ and $\nabla$ are flat logarithmic connections, the natural connection $\nabla^{\prime \prime}$ on $\mathcal{E}^{\prime \prime}$ is clearly logarithmic. According to the classical Riemann-Hilbert correspondence, the isomorphism $\psi_{0}$ between the initial connections extends in a unique way to an isomorphism $\psi:\left.\left.\left(\mathcal{E}^{\prime}, \nabla^{\prime}\right)\right|_{\mathcal{X}_{\mathcal{T}}^{*}} \stackrel{\sim}{\longrightarrow}(\mathcal{E}, \nabla)\right|_{\mathcal{X}_{\mathcal{T}}^{*}}$ over $\mathcal{X}_{\mathcal{T}} \backslash \mathcal{D}_{\mathcal{T}}$. This isomorphism can be seen as a horizontal section of $\left.\left(\mathcal{E}^{\prime \prime}, \nabla^{\prime \prime}\right)\right|_{\mathcal{V}^{i} \backslash \mathcal{D}_{\mathcal{T}}^{i}}$, where $\mathcal{V}^{i}$ is a small neighborhood of $D_{0}^{i}$ in $\mathcal{X}_{\mathcal{T}}$. In particular, the section $\psi$ is uniform, i.e. it has no monodromy on $\mathcal{V}^{i} \backslash \mathcal{D}_{\mathcal{T}}^{i}$. On the one hand, the fact that $\nabla^{\prime \prime}$ is a flat logarithmic connection (and thus $\left.\nabla^{\prime \prime}\right|_{\mathcal{V}^{i} \backslash \mathcal{D}_{\mathcal{T}}^{i}}$ is regular singular) implies that this uniform section $\psi$ can be continued analytically at $\mathcal{D}_{\mathcal{T}}^{i}$. It is clear from the Cauchy theorem that the zero set of a horizontal section of a flat logarithmic connection is either trivial, or it defines a divisor of codimension 1 on the base curve which has to be a subset of the polar divisor, i.e. it is equal to the polar divisor. By consequence, the analytic continuation of our horizontal section $\psi$ on $\mathcal{V}^{i} \backslash \mathcal{D}_{\mathcal{T}}^{i}$ to $\mathcal{V}^{i}$ is 
either holomorphic on $\mathcal{V}^{i} \cap \mathcal{D}_{\mathcal{T}}^{i}$, or for each $\tau_{1} \in \mathcal{T}$ it has a pole on $\mathcal{V}^{i} \cap\left\{\tau=\tau_{1}\right\}$. On the other hand, the restriction $\psi_{0}$ of $\psi$ at $X_{0}$ is holomorphic at $D_{0}^{i}$. Thus $\psi$ is holomorphic over $\mathcal{V}^{i}$ and can be continued holomorphically along $\mathcal{D}_{\mathcal{T}}^{i}$. In using the same argument for $\psi^{-1}$, we see that $\left(\mathcal{E}^{\prime}, \nabla^{\prime}\right)$ and $(\mathcal{E}, \nabla)$ can be identified over $\mathcal{X}_{\mathcal{T}}$ by means of a unique isomorphism which extends $\psi_{0}$.

Remark 8. It is clear from the upper proof that the polar set of a flat logarithmic connection on a family of marked Riemann surfaces is closed. Moreover, if the polar divisor is contractible and transverse to the parameter (as in our case), it follows from proposition 1 that such a flat logarithmic connection is locally constant in the $\mathfrak{s l}(2, \mathbf{C})$-case.

\subsection{General case}

We are now going to construct the universal isomonodromic deformation in the general meromorphic $\mathfrak{s l}(2, \mathbf{C})$-case. Like in the logarithmic case, we get a non-singular connection $\left(\mathcal{E}^{*}, \nabla^{*}\right)$ over the punctured universal curve $\mathcal{X}_{\mathcal{T}}^{*}$ over the Teichmüller space $\mathcal{T}=$ Teich $(g, m)$, which is unique up to unique isomorphisms. We may also construct local connections $\left(\mathcal{E}^{i}, \nabla^{i}\right)$ over germs of tubular neighborhoods $\mathcal{U}_{\mathcal{T}}^{i}$ of $\mathcal{D}_{\mathcal{T}}^{i}$ in order to stuff the gaps. These are unique only up to gauge- and coordinate-transformations. Again, both connections are non-singular over the punctured neighborhood $\mathcal{U}_{\mathcal{T}}^{i}{ }^{*}=\mathcal{U}_{\mathcal{T}}^{i} \backslash \mathcal{D}_{\mathcal{T}}^{i}$ and they have the same local monodromy. The classical Riemann-Hilbert correspondence thus provides an isomorphism gluing $\left(\mathcal{E}^{*}, \nabla^{*}\right)$ and the $\left(\mathcal{E}^{i}, \nabla^{i}\right)$ into a connection over $\mathcal{X}_{\mathcal{T}}$. Yet the gluing will not be unique in general : it depends on the chosen gluing of the coordinates on the germ $\mathcal{U}_{\mathcal{T}}^{i}$. Thereby we get additional parameters in the construction.

\subsubsection{Freedom in the gluing construction}

Let us now see in detail why the resulting connection might not be unique. Since both $\left(\mathcal{E}^{*}, \nabla^{*}\right)$ and $\left(\mathcal{E}^{i}, \nabla^{i}\right)$ are constant in appropriate local coordinates, it makes sense to consider firstly a similar situation without parameter (and forget the condition of extending the initial connection for a while). In spite of the tublar neighborhood $\mathcal{U}_{\mathcal{T}}^{i}$, let us consider a germ $(\mathbf{C}, 0)$. Let $\nabla^{i}$ be a connection on $E^{i}=(\mathbf{C}, 0) \times \mathbf{C}^{2}$ given in coordinates $(x, Y)$ by $\mathrm{dY}=A(x) Y \mathrm{~d} x$ with a pole at $\{x=0\}$. Let $\left(E^{*}, \nabla^{*}\right)$ be a non-singular connection over $\left(\mathbf{C}^{*}, 0\right)$ and suppose there is a gluing isomorphism between the two connections. Let $V \times \mathbf{C}^{2}$ be a simply connected chart of $E^{*}$ where $\nabla^{*}$ is given by $\mathrm{d} Y=0$. Denote by $Y \mapsto \phi\left(x_{i}\right) Y$ be the gauge transformation on $\mathcal{V} \times \mathbf{C}^{2}$ defined by the restriction on $V$ of the gluing isomorphism from $\left(E^{i}, \nabla^{i}\right)$ to $\left(E^{*}, \nabla^{*}\right)$. Let $\varphi$ be any diffeomorphism of the germ $(\mathbf{C}, 0)$. Now the gauge-coordinate transformation $(\varphi(x), \phi(x) Y)$ will also conjugate the two systems. We thus have constructed another gluing isomorphism on $\left(\mathbf{C}^{*}, 0\right)$. 


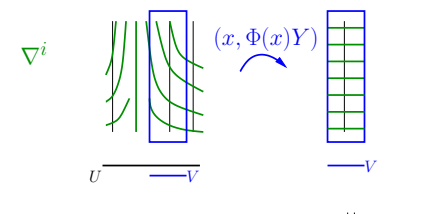

$\nabla^{*}$

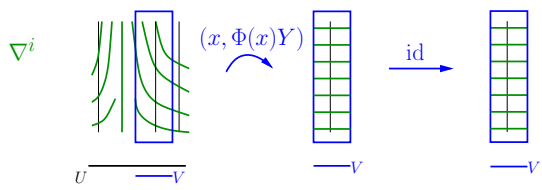

|| gluing 2

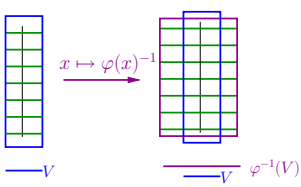

We will get the same connection for this second gluing if, and only if, $\varphi^{*} \nabla^{i}$, given by the system $\mathrm{dY}=A \circ \varphi^{-1}(x) Y \mathrm{~d} \varphi^{-1}(x)$, is conjugated to $\nabla^{i}$, given by the system $\mathrm{dY}=$ $A(x) Y \mathrm{~d} x$, by a holomorphic gauge transformation $Y \mapsto \widetilde{\phi}(x) Y$ :

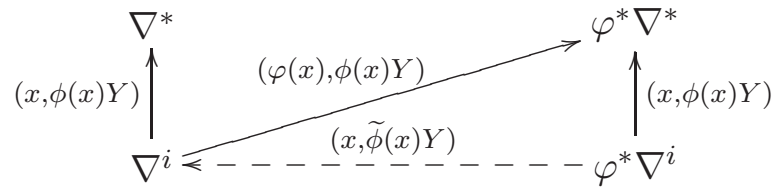

Or equivalently, we get the same gluing construction if there is a holomorphic gauge transformation $\widetilde{\phi}(x)$ such that $(\varphi(x), \widetilde{\phi}(\varphi(x)) Y)$ is conjugating $\nabla^{i}$ to itself :

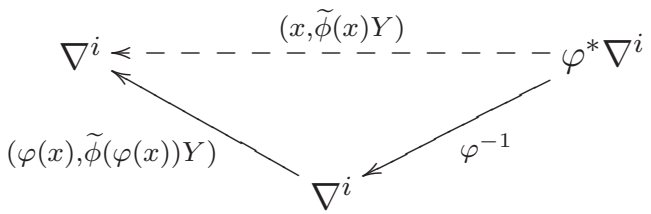

We saw that there is a unique connection resulting from such a gluing in the logarithmic case. In the general case, we have the following result.

Lemma 2. Let $\nabla^{i}$ be a connection on the trivial vector bundle $E^{i}$ over a germ $(\mathbf{C}, 0)$ with coordinate $x$, having a pole of order $l$ at $\{x=0\}$. Let $\varphi(x)$ be a holomorphic diffeomorphism of $(\mathbf{C}, 0)$ such that

$$
\varphi(x) \equiv \operatorname{id}(x) \quad \bmod x^{l} .
$$

Then there is a holomorphic gauge transformation $\widetilde{\phi}(x)$ such that $(\varphi(x), \widetilde{\phi}(\varphi(x)) Y)$ is conjugating $\nabla^{i}$ to itself.

Proof: Choose coordinates $(x, Y)$ such that $\left(E^{i}, \nabla^{i}\right)$ is given by $\mathrm{d} Y=A(x) Y \mathrm{~d} x$ with connection matrix

$$
A(x)=\frac{1}{x^{l}}\left(\begin{array}{cc}
a(x) & b(x) \\
c(x) & -a(x)
\end{array}\right)
$$


which has a pole of order $l$. In order to prove this lemma, we shall use the path method of J. Moser (see [Mos65], see also appendix 1 in [DZ05]). Our starting point is the connection $\left(E_{0}^{i}, \nabla_{0}^{i}\right)=\left(E^{i}, \nabla^{i}\right)$ on $(\mathbf{C}, 0)$. We want to prove that it is conjugated to the connection $\left(E_{1}^{i}, \nabla_{1}^{i}\right)=\varphi^{*}\left(E^{i}, \nabla^{i}\right)$ on $(\mathbf{C}, 0)$ by a gauge-transformation $\widetilde{\phi}$. By $\left(\varphi_{s}(x)\right)_{s \in[0,1]}$ with $\varphi_{s}(x)=s \varphi(x)+(1-s) \operatorname{id}(x)$, we define an analytic isotopy of holomorphic diffeomorphisms on $(\mathbf{C}, 0)$ joining $\varphi$ to the identity. It defines an analytic path of connections $\left(E_{s}^{i}, \nabla_{s}^{i}\right)_{s \in[0,1]}$ by $\left(E_{s}^{i}, \nabla_{s}^{i}\right)=\varphi_{s}^{*}\left(E^{i}, \nabla^{i}\right)$. We want to find an analytic path of gauge-transformations $\left(\phi_{s}(x)\right)_{s \in[0,1]}$ such that $\left(E_{s}^{i}, \nabla_{s}^{i}\right)$ is conjugated to $\left(E_{0}^{i}, \nabla_{0}^{i}\right)$ by $\phi_{s}$. Note that the analytic isotopy of diffeomorphisms $\left(\varphi_{s}(x)\right)_{s \in[0,1]}$ defines the flow of a vector field $v(s, x)$ such that $v\left(s, \varphi_{s}(x)\right)=\frac{\partial}{\partial s}+\left[\frac{\partial}{\partial s} \varphi_{s}(x)\right] \frac{\partial}{\partial x}$. Indeed, $v$ is given by

$$
v(s, x)=\frac{\partial}{\partial s}+\varphi_{s}^{*} v_{0}(x),
$$

where $v_{0}(x)=(\varphi(x)-x) \frac{\partial}{\partial x}$.

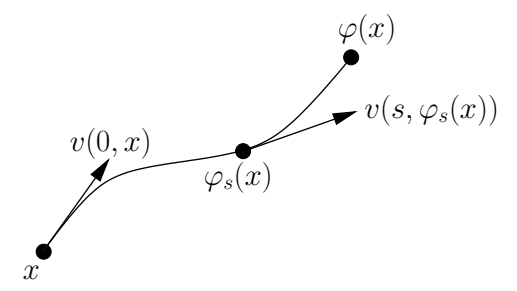

Figure 1: Vector field $v$ associated to the isotopy of diffeomorphisms

By assumption, $v_{0}$ has a zero of order $l$ at $x=0$. Then

$$
v(s, x)=\frac{\partial}{\partial s}+x^{l} f_{s}(x) \frac{\partial}{\partial x},
$$

where $\left(f_{s}\right)_{s \in[0,1]}$ is an analytic isotopy of holomorphic functions. Recall that the connection matrix $A$ of the initial connection has a pole of order $l$. By consequence, a holomorphic vector field on $E_{0}^{i}$ tangent to the connection $\nabla_{0}^{i}$ is a holomorphic multiple of

$$
\mathcal{W}=x^{l} \frac{\partial}{\partial x}+\left(a(x) y_{1}+b(x) y_{2}\right) \frac{\partial}{\partial y_{1}}+\left(c(x) y_{1}-a(x) y_{2}\right) \frac{\partial}{\partial y_{2}} .
$$

By (6), for each time $s_{1} \in[0,1]$, the vector field $\left.v\right|_{s=s_{1}}$ thus lifts to the vector field $\mathcal{V}_{s_{1}}=$ $f_{s}(x) \mathcal{W}$ on $E_{0}^{i}$, which is tangent to the connection $\nabla_{0}^{i}$. The flow of the vector field

$$
\mathcal{V}(s, x)=\frac{\partial}{\partial s}+f_{s}(x) \mathcal{W}
$$

defines an analytic isotopy of holomorphic gauge-coordinate-transformations of the form

$$
\left(\Phi_{s}^{\mathcal{V}}\right)_{s \in[0,1]}=\left(\varphi_{s}, \phi_{s}\right)_{s \in[0,1]}
$$


They are indeed gauge-transformations with respect to the $Y$-coordinate, since we are integrating in the Lie-algebra of gauge-transformations. By construction, we have $\left(\Phi_{s}^{\mathcal{V}}\right)^{*}\left(E_{0}^{i}, \nabla_{0}^{i}\right)=$ $\left(E_{0}^{i}, \nabla_{0}^{i}\right)$ for each time $s \in[0,1]$. In other words, for each time $s \in[0,1]$, the gaugetransformation $\widetilde{\phi}_{s}=\varphi_{s}^{*} \phi_{s}$ then conjugates the connection $\left(E_{s}^{i}, \nabla_{s}^{i}\right)$ to $\left(E_{0}^{i}, \nabla_{0}^{i}\right)$, as desired. In particular, we have found a gauge-coordinate transformation of the form

$$
\Phi_{1}^{\mathcal{V}}(x, Y)=(\varphi(x), \phi(x) Y)=(\varphi(x), \widetilde{\phi}(\varphi(x)) Y)
$$

such that $\left(\Phi_{1}^{\mathcal{V}}\right)^{*}\left(E_{0}^{i}, \nabla_{0}^{i}\right)=\left(E_{0}^{i}, \nabla_{0}^{i}\right)$.

Inversely, if there is an analytic isotopy $\left(\varphi_{s}(x), \phi_{s}(x) Y\right)_{s \in[0,1]}$ with $\left(\varphi_{0}, \phi_{0}\right)=(\mathrm{id}, I)$ of holomorphic gauge-coordinate transformations on $E^{i}$ keeping $\nabla^{i}$ invariant, then we can associate a vector field depending on $s$ tangent to the connection, as we will see in lemma 4. This implies $\varphi_{s}(x) \equiv \operatorname{id}(x) \bmod x^{l}$.

Remark 9. It is possible that there are gauge-coordinate transformations on $E^{i}$ keeping $\nabla^{i}$ invariant and such that the induced coordinate change is not tangent to the identity. For example $\mathrm{d} Y=\frac{1}{x^{l}} Y \mathrm{~d} x$ is invariant under the coordinate transformation $x \mapsto e^{\frac{2 i \pi}{l-1}} x$. But the set of such coordinate transformations is discrete.

\subsubsection{Explicit construction}

In order to construct the universal isomonodromic deformation in the case of multiple poles, consider for each pole of order $n_{i}>1$ the set $J^{i}=\operatorname{Jets}^{<n_{i}}(\operatorname{Diff}(\mathbf{C}, 0))$ of $\left(n_{i}-1\right)$-jets of biholomorphisms of $(\mathbf{C}, 0)$. We identify $J^{i}$ to $\mathbf{C}^{*} \times \mathbf{C}^{n_{i}-2}$, where $s=\left(s_{1}, \ldots, s_{n_{i}-1}\right)$ is associated to $\varphi_{s}(x)=s_{1} x+s_{2} x^{2}+\ldots+s_{n_{i}-1} x^{n_{i}-1}$. For a simple pole $x_{j}=0$ we may consider $J^{j}$ being the singleton of the identity. Let $J$ be the universal cover $J=\widetilde{J^{1}} \times \ldots \times \widetilde{J^{m}}$ of the space of jets, where $\widetilde{J}^{i}=\widetilde{\mathbf{C}^{*}} \times \mathbf{C}^{n_{i}-2}$. Our parameter space $T$ for the universal isomonodromic deformation will be

$$
T=J \times \mathcal{T} .
$$

Remark 10. This parameter space is contractible. In particular, we avoid monodromy phenomena along the parameter space.

Our universal curve $(\mathcal{X}, \mathcal{D}) \rightarrow T$ shall be the product of the the space of jets $J$ with the universal Teichmüller curve :

$$
(\mathcal{X}, \mathcal{D})=\left(J \times \mathcal{X}_{\mathcal{T}}, J \times \mathcal{D}_{\mathcal{T}}\right)
$$

This curve will be the base curve of the universal isomonodromic deformation of $\left(E_{0} \rightarrow\right.$ $\left.X_{0}, \nabla_{0}\right)$. As before, denote by $\mathcal{X}^{*}$ the universal curve minus the distinguished points and 
their deformations $\mathcal{D}^{i}=J \times \mathcal{D}_{\mathcal{T}}^{i}$. Let $\nabla^{*}$ be the unique non-singular integrable connection over $\mathcal{X}^{*}$ having monodromy $\rho$. For $t_{0}=\left((\mathrm{id}, \ldots, \mathrm{id}), \tau_{0}\right) \in T$, we have $\left.\nabla^{*}\right|_{t=t_{0}}=\nabla_{0}^{*}$.

We have again tubular neighborhoods $\mathcal{U}^{i}=J \times \mathcal{U}_{\mathcal{T}}^{i}$ of $\mathcal{D}^{i}$ on $\mathcal{X}$ and functions $\xi_{i}: \mathcal{U}^{i} \rightarrow \mathbf{C}$, identical to the Teichmüller coordinate, but seen on the bigger space, satisfying $\mathcal{D}^{i}=$ $\operatorname{div}\left(\xi_{i}\right)$. On $U_{0}^{i}=\left.\mathcal{U}^{i}\right|_{t=t_{0}}$ with coordinate $x_{i}$, the initial connection induces a local connection $\nabla_{0}^{i}$ on the trivial bundle $E_{0}^{i}=U_{0}^{i} \times \mathbf{C}^{2}$ defined by a system

$$
\nabla_{0}^{i}: \quad \mathrm{d} Y=A\left(x_{i}\right) Y \mathrm{~d} x_{i}
$$

Then on the tubular neighborhood $\mathcal{U}^{i}$, we define an integrable, locally constant connection $\nabla^{i}$ on the trivial bundle $\mathcal{E}^{i}=\mathcal{U}^{i} \times \mathbf{C}^{2}$ with polar set $\mathcal{D}^{i}$. Firstly, we define the connection

$$
\nabla_{0}^{i}: \quad \mathrm{d} Y=A\left(\xi_{i}\right) Y \mathrm{~d} \xi_{i}
$$

on the trivial vector bundle $\mathcal{E}_{0}^{i}$ over $\mathcal{U}^{i}=J \times \mathcal{U}_{\mathcal{T}}^{i}$ as a product from the initial connection $\nabla_{0}^{i}:$

$$
\left(\mathcal{E}_{0}^{i}, \nabla_{0}^{i}\right)=\xi_{i}^{*}\left(E_{0}^{i}, \nabla_{0}^{i}\right)
$$

Then for coordinates $\left(\varphi_{1}, \ldots, \varphi_{m}\right) \in J=\prod_{i=1}^{m} \widetilde{J}^{i}$, we define $\nabla^{i}$ on the trivial vector bundle $\mathcal{E}^{i}$ over $\mathcal{U}^{i}$ by $\left(\mathcal{E}^{i}, \nabla^{i}\right)=\varphi_{i}^{*}\left(\mathcal{E}_{0}^{i}, \widetilde{\nabla}_{0}^{i}\right)$. Namely, we define

$$
\nabla^{i}: \quad \mathrm{d} Y=A\left(\left(\varphi_{i}\right)^{-1}\left(\xi_{i}\right)\right) Y \mathrm{~d}\left(\left(\varphi_{i}\right)^{-1}\left(\xi_{i}\right)\right) .
$$

We then have $\left(E_{0}^{i}, \nabla_{0}^{i}\right)=\left.\left(\mathcal{E}^{i}, \nabla^{i}\right)\right|_{t=t_{0}}$. We now have to glue $\left(\mathcal{E}^{i}, \nabla^{i}\right)$ with $\left(\mathcal{E}^{*}, \nabla^{*}\right)$. Let $\psi_{0}^{i}$ be the gluing isomorphism from $\left(E_{0}^{*}, \nabla_{0}^{*}\right)$ to $\left(E_{0}^{i}, \nabla_{0}^{i}\right)$.

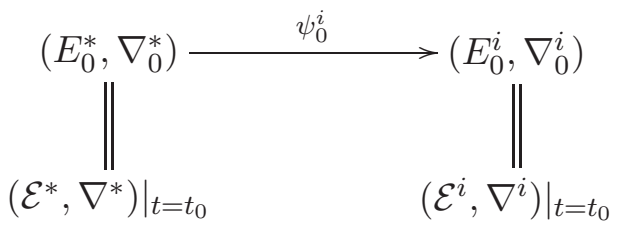

On the base curve, we choose the natural, i.e. the identity gluing from $\mathcal{X}^{*}$ to $\mathcal{U}^{i}$ on the intersection $\mathcal{U}^{i^{*}}=\mathcal{U}^{i} \backslash \mathcal{D}^{i}$. According to the classical Riemann-Hilbert correspondence and its corollary, there is a gluing isomorphism $\psi^{i}$ given by gauge transformations over $\mathcal{U}^{i^{*}}$ from $\left(\mathcal{E}^{*}, \nabla^{*}\right)$ to $\left(\mathcal{E}^{i}, \nabla^{i}\right)$ and which extends the initial gluing isomorphism :

$$
\left.\psi^{i}\right|_{t=t_{0}}=\psi_{0}^{i}
$$

Gluing in this manner each of the local connections $\left(\mathcal{E}^{i}, \nabla^{i}\right)$ with $\left(\mathcal{E}^{*}, \nabla^{*}\right)$, we have constructed a flat integrable, locally constant tracefree connection $(\mathcal{E} \rightarrow \mathcal{X}, \nabla)$ over $\mathcal{X} \rightarrow T$ with polar set $\mathcal{D}=\sum_{i=1}^{m} \mathcal{D}^{i}$ which satisfies

$$
\left.(\mathcal{E} \rightarrow \mathcal{X}, \nabla)\right|_{t=t_{0}}=\left(E_{0} \rightarrow X_{0}, \nabla_{0}\right) .
$$


We remark that the so-defined universal isomonodromic deformation is global in reference to the Teichmüller space as well as in reference to the space of jets.

If the Teichmüller space $\mathcal{T}$ has dimension $3 g-3+m \geq 0$, then the dimension of the parameter space $T$ of the universal isomonodromic deformation constructed above is $3 g-3+m+\sum_{i=1}^{m}\left(n_{i}-1\right)$. Thus

$$
\operatorname{dim}(T)=3 g-3+n
$$

\section{Proof of the universal Property}

Let us now prove the universal property theorem 1 for the above constructed universal isomonodromic deformation $(\mathcal{E} \rightarrow \mathcal{X}, \nabla)$ over $\mathcal{X} \rightarrow T$ with polar set $\mathcal{D}$. Let $(\widetilde{E} \rightarrow \tilde{X}, \widetilde{\nabla})$ be another isomonodromic deformation of the initial connection. Let $n$ (resp. $m$ ) be the number of poles counted with (resp. without) multiplicity, as before. We will denote by $\sum_{i=1}^{m} n_{i} \widetilde{\mathcal{D}}^{i}$ (resp. $\widetilde{\mathcal{D}}$ ) the divisor (resp. the reduced divisor) of $(\widetilde{E} \rightarrow \widetilde{X}, \widetilde{\nabla})$. The reduced divisor of $\nabla_{0}$ will be denoted by $D_{0}$, as usual. Using the product structure of the parameter space $T=J \times \mathcal{T}$, we will construct holomorphic maps $(f, F)$ extending the initial isomorphism $\left(f_{0}, F_{0}\right)$ of marked Riemann surfaces, such that the diagram

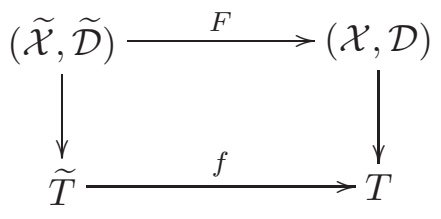

commutes and such that in restriction to each parameter $\tilde{t} \in \widetilde{T}$, the map $F$ induces an isomorphism of marked Riemann surfaces. Therefore we will firstly consider the universal curve respective to the deformation in the Teichmüller space, secondly the deformation respective to the jets. Afterwards we will define an isomorphism $\psi:(\widetilde{\mathcal{E}}, \widetilde{\nabla}) \stackrel{\sim}{\rightarrow} F^{*}(\mathcal{E}, \nabla)$, which extends the given isomorphism $\psi_{0}:\left(\widetilde{E}_{0}, \widetilde{\nabla}_{0}\right) \stackrel{\sim}{\rightarrow} F_{0}^{*}\left(E_{0}, \nabla_{0}\right)$. Here $\psi\left(\right.$ resp. $\left.\psi_{0}\right)$ are isomorphisms between connections on the same base curve $\widetilde{\mathcal{X}},\left(\right.$ resp. $\left.\widetilde{X}_{0}\right)$ and will thus be given by local gauge transformations. The maps $\Psi$ (resp. $\left.\Psi_{0}\right)$ then are obtained via $F$ (resp. $\left.F_{0}\right)$. The triple $(f, F, \Psi)$ will be unique if $(g, m)$ is different from $(0,0),(0,1),(0,2)$ and $(1,0)$. Finally we will study the default of uniqueness in the special cases.

\subsection{Classifying map and lift to the base curve}

Denote again by $\mathcal{X}_{\mathcal{T}} \rightarrow \mathcal{T}$ the universal Teichmüller curve respective to $X_{0}$, and by $\mathcal{X} \rightarrow T$ the universal curve underlying the universal isomonodromic deformation of $\left(E_{0} \rightarrow X_{0}, \nabla_{0}\right)$. Consider the Teichmüller classifying map $h$ from $\left(\widetilde{T}, \tilde{t}_{0}\right)$ to $\left(\mathcal{T}, \tau_{0}\right)$. The map $h$ is holomor- 
phic and induces a holomorphic map $H$, making the following diagramm commute

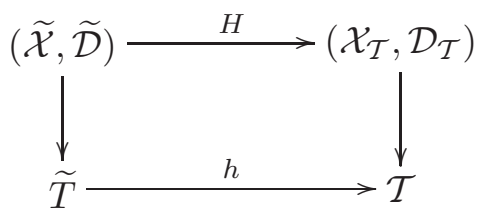

and such that $H$ defines an isomorphism of marked Riemann surfaces in each fibre (see [Nag88], page 349).

Remark 11. The cases $g=0, m=1,2$ are not explicitly treated in [Nag88], but the result remains true in our context since any analytic fibre space whose fibres are all holomorphically equivalent to one compact connected complex manifold is locally trivial (see [FG65]).

From the tubular neighborhoods $\mathcal{U}_{\mathcal{T}}^{i}$ in the Teichmüller curve, via $F$ we get tubular neighborhoods $\widetilde{\xi}_{i}: \widetilde{\mathcal{U}}^{i} \rightarrow \mathbf{C}$ of $\widetilde{\mathcal{D}}^{i}$ and thus the connection $\left(\widetilde{E}_{0}^{i}, \widetilde{\nabla}_{0}^{i}\right)$, induced by the initial connection $\left(\widetilde{E}_{0}, \widetilde{\nabla}_{0}\right)$ on $\widetilde{\mathcal{U}}^{i} \cap X_{0}$, can be considered as a product connection on $\widetilde{\mathcal{U}}^{i}$. We will now construct a classifying map in the space of local jets. From the point of view of the product connection $\left(\widetilde{\mathcal{E}}_{0}^{i}, \widetilde{\nabla}_{0}^{i}\right)=\widetilde{\xi}_{i}^{*}\left(\widetilde{E}_{0}^{i}, \widetilde{\nabla}_{0}^{i}\right)$, the gluing isomorphism between $\left(\widetilde{\mathcal{E}}^{i}, \widetilde{\nabla}^{i}\right)$ and $\left(\widetilde{\mathcal{E}}^{*}, \widetilde{\nabla}{ }^{*}\right)=\left.(\widetilde{\mathcal{E}}, \widetilde{\nabla})\right|_{\widetilde{X}^{*}}$ will only depend on the gluing of $\widetilde{\mathcal{U}}^{i}$ to $\widetilde{X}^{*}$ in the base curve. From now on, we will forget the variable $Y$ in the sense that we only indicate whether or not there are gauge-transformations conjugating two given connections.

Let $\left(\tilde{t}, x_{i}\right)$ be a local coordinate on $\widetilde{\mathcal{U}}^{i}$ defined in a small neighborhood of $\tilde{t}_{0}$. Assume that $\widetilde{\mathcal{D}}^{i}$ is given in these coordinates by $\left\{x_{i}=0\right\}$.

Lemma 3. There is a coordinate-transformation $\left(\tilde{t}, x_{i}\right) \mapsto\left(\tilde{t}, \tilde{\varphi}_{i}\left(\tilde{t}, x_{i}\right)\right)$ fixing $\widetilde{\mathcal{D}}^{i}$ such that locally on the considered open set of $\widetilde{\mathcal{U}}^{i}$, we have

$$
\left\{\begin{array}{l}
\mathrm{id}=\left.\tilde{\varphi}_{i}\right|_{\tilde{t}=\tilde{t}_{0}} \\
\widetilde{\nabla}^{i}=\left(\tilde{\varphi}_{i}\right)^{*} \widetilde{\nabla}_{0}^{i},
\end{array}\right.
$$

where $\widetilde{\nabla}_{0}^{i}$ is considered as a connection on the trivial bundle $\widetilde{\mathcal{E}}_{0}^{i}=\widetilde{E}_{0}^{i} \times \widetilde{T}$ over $\widetilde{\mathcal{U}}^{i}$.

Proof: According to the local constancy, there are local gauge-coordinate transformations $\left(\tilde{t}, x_{i}, Y_{i}\right) \mapsto\left(\tilde{t}, \varphi_{i}\left(\tilde{t}, x_{i}\right), \phi_{i}\left(\tilde{t}, x_{i}\right) \cdot Y_{i}\right)$ trivializing the connection $\widetilde{\nabla}^{i}$ in the parameter $\tilde{t}$ and fixing $\widetilde{\mathcal{D}}_{i}$. Then

$$
\left(\tilde{t}, x_{i}, Y_{i}\right) \mapsto\left(\tilde{t},\left(\varphi_{i}\right)^{-1}\left(\tilde{t}_{0}, \varphi_{i}\left(\tilde{t}, x_{i}\right)\right),\left(\phi_{i}\right)^{-1}\left(\tilde{t}_{0}, x_{i}\right) \cdot \phi_{i}\left(\tilde{t}, x_{i}\right) \cdot Y_{i}\right)
$$

satisfies the conditions $(7)$.

Now let us consider $\widetilde{\varphi}_{i}\left(\tilde{t}, x_{i}\right)$ as a holomorphic family of holomorphic diffeomorphisms $\tilde{\varphi}_{i}^{\tilde{t}}\left(x_{i}\right)$ of $(\mathbf{C}, 0)$. 
Lemma 4. Let $\widetilde{\varphi}_{i}^{\tilde{t}}$ and $\widetilde{\widetilde{\varphi}}_{i}^{\tilde{t}}$ be two holomorphic families of biholomorphisms satisfying (7). Then they are equivalent modulo $x_{i}^{n_{i}}$.

Proof: Consider the biholomorphism $\varphi_{\tilde{t}}=\left(\widetilde{\widetilde{\varphi}}^{\tilde{t}}\right)^{-1} \circ \tilde{\varphi}_{i}^{\tilde{t}}$. Then $\varphi_{\tilde{t}}^{*} \widetilde{\nabla}_{0}^{i}=\widetilde{\nabla}_{0}^{i}$ for each parameter $\tilde{t} \in \widetilde{T}$. This means there is a gauge transformation $Y_{i} \mapsto \phi_{\tilde{t}}\left(x_{i}\right) \cdot Y_{i}$ such that the gauge-coordinate-transformation $\left(\varphi_{\tilde{t}}, \phi_{\tilde{t}}\right)$ conjugates the system

$$
\mathrm{d} Y_{i}=\frac{1}{x_{i}^{n_{i}}} A\left(x_{i}\right) Y_{i} \mathrm{~d} x_{i}
$$

defining $\widetilde{\nabla}_{0}^{i}$ to itself.

Let us now fix a parameter $\tilde{t}_{1} \in \widetilde{T}$. Let $\gamma:[0,1] \rightarrow \widetilde{T}$ be an analytic path with $\gamma(0)=\tilde{t}_{0}, \gamma(1)=\tilde{t}_{1}$. Then $\left(\varphi_{\gamma(s)}, \phi_{\gamma(s)}\right)_{s \in[0,1]}$ is an analytic isotopy of gauge-coordinatetransformations keeping $\widetilde{\nabla}_{0}^{i}$ invariant. Moreover, this isotopy contains (id, $I$ ) for the initial parameter $s=0$. As in lemma 2, we may associate to this isotopy the vector field

$$
\mathcal{V}\left(s, \varphi_{\gamma(s)}\left(x_{i}\right)\right)=\frac{\partial}{\partial s}+\left[\frac{\partial}{\partial s}\left(\varphi_{\gamma(s)}, \phi_{\gamma(s)}\right)\right]
$$

which is tangent to the connection for each time $s=s_{1} \in[0,1]$. We may also consider the vector field $v\left(s, \varphi_{\gamma(s)}\left(x_{i}\right)\right)=\frac{\partial}{\partial s}+\left[\frac{\partial}{\partial s} \varphi_{\gamma(s)}\left(x_{i}\right)\right]$ on the base curve. By construction, for each time $s_{1} \in[0,1]$, the vector field $\left.v\right|_{s=s_{1}}$ lifts to the holomorphic vector field $\left.\mathcal{V}\right|_{s=s_{1}}=$ $\left.v\right|_{s=s_{1}}+f\left(s_{1}, x_{i}\right) A Y_{i}$, which has to be tangent to the connection $\widetilde{\nabla}_{0}^{i}$.

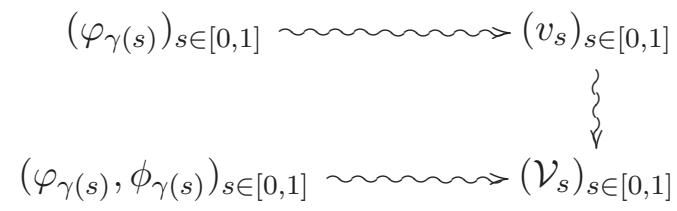

By consequence, $\left.v\left(s, \varphi_{\gamma(s)}\left(x_{i}\right)\right)\right|_{s=s_{1}}$ has to be zero modulo $x_{i}^{n_{i}}$ for each $s_{1} \in[0,1]$. Since $v$ is analytic and $\varphi_{\gamma(0)}$ is the identity, it follows that $\varphi_{\gamma(s)}$ is equal to the identity modulo $x_{i}^{n_{i}}$. In particular, $\varphi_{\tilde{t}_{1}}=\mathrm{id} \bmod x_{i}^{n_{i}}$.

Inversely, if $\tilde{\varphi}_{i}$ is satisfying (7) and $\tilde{\tilde{\varphi}}_{i}$ is a holomorphic family of diffeomorphisms with

$$
\tilde{\varphi}_{i} \equiv \tilde{\varphi}_{i} \bmod x_{i}^{n_{i}},
$$

then $\tilde{\tilde{\varphi}}_{i}$ also satisfies (7), according to lemma 2 .

For each parameter $\tilde{t}$ in a neighborhood $W_{0}$ of the initial parameter $\tilde{t}_{0}$ in $\widetilde{T}$, we can find a biholomorphism $\tilde{\varphi}_{i}\left(\tilde{t}, x_{i}\right)$ as in lemma 4 , whose $\left(n_{i}-1\right)$-jet is uniquely defined according to lemma 4 . The map associating to a parameter in $\widetilde{T}$ the associated $\left(n_{i}-1\right)$-jet of 
diffeomorphisms can be analytically continued along any path in $\widetilde{T}$. Indeed, choose an open set $W_{1}$ in the germ $\widetilde{\mathcal{U}}^{i}$, such that the connection $\widetilde{\nabla}^{i}$ is locally trivial on this open set up to a convenient gauge-coordinate transformation and such that $W_{0} \cap W_{1} \neq \emptyset$. Choose a parameter $t_{1} \in W_{0} \cap W_{1}$, and let $\tilde{\varphi}_{t_{1}}$ be the associated diffeomorphism. Recall that $\widetilde{\nabla}_{0}^{i}$ can be seen naturally as a connection on $\widetilde{\mathcal{U}}^{i}$. Like in the above lemmas, we see that on $W_{1}$, there is a family of diffeomorphisms $\tilde{\varphi}_{i}$ such that

$$
\left\{\begin{array}{l}
\tilde{\varphi}_{t_{1}}=\left.\tilde{\varphi}_{i}\right|_{\tilde{t}=\tilde{t}_{1}} \\
\widetilde{\nabla}^{i}=\left(\tilde{\varphi}_{i}\right)^{*} \widetilde{\nabla}_{0}^{i},
\end{array}\right.
$$

and the $\left(n_{i}-1\right)$-jet of this family is unique. In particular, it continues analytically the family of diffeomorphisms on $W_{0}$. Since $\widetilde{T}$ is contractible, we can associate to each parameter $\tilde{t} \in \widetilde{T}$ a biholomorphism $\tilde{\varphi}_{i}\left(\tilde{t}, x_{i}\right)$ by analytic continuation, such that the following map is well-defined and holomorphic:

$$
g^{i}: \begin{aligned}
\widetilde{T} & \longrightarrow J^{i} \\
\tilde{\varphi}_{i} & \longmapsto \tilde{\varphi}_{i} \bmod x_{i}^{n_{i}} .
\end{aligned}
$$

The map

$$
g:\left(\widetilde{T}, \tilde{t}_{0}\right) \stackrel{\left(g^{1}, \ldots, g^{m}\right)}{\longrightarrow}\left(J^{1} \times \ldots \times J^{m},(\mathrm{id}, \ldots, \mathrm{id})\right)
$$

constructed in that way can be lifted to the universal cover $J=\widetilde{J^{1}} \times \ldots \times \widetilde{J^{m}}$. Finally, denote by $G$ the trivial lift

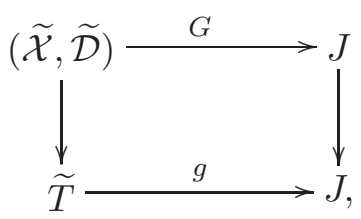

mapping fibres to singletons. Since $T=J \times \mathcal{T}$, we obtain a canonical holomorphic mapping

$$
f:\left(\widetilde{T}, \tilde{t}_{0}\right) \stackrel{(g, h)}{\longrightarrow}\left(T, t_{0}\right),
$$

where $t_{0}=\left((\mathrm{id}, \ldots, \mathrm{id}), \tau_{0}\right)$. Recall that the universal curve $\mathcal{X}$ is constructed as a product $(\mathcal{X}, \mathcal{D})=\left(J \times \mathcal{X}_{\mathcal{T}}, J \times \mathcal{D}_{\mathcal{T}}\right)$. The Teichmüller map $H: \widetilde{\mathcal{X}} \rightarrow \mathcal{X}_{\mathcal{T}}$ thus lifts via $G$ to a holomorphic map $F:(\widetilde{\mathcal{X}}, \widetilde{\mathcal{D}}) \rightarrow(\mathcal{X}, \mathcal{D})$, such that

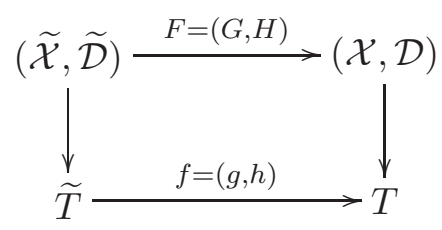

commutes and $F$ defines an isomorphism of marked Riemann surfaces in each fibre. Moreover, the maps $(f, F)$ are extending $\left(f_{0}, F_{0}\right)$, by construction. 


\subsection{Lift to an isomorphism of connections}

We want to extend the initial isomorphism $\psi_{0}:\left(\widetilde{E}_{0}, \widetilde{\nabla}_{0}\right) \rightarrow F_{0}^{*}\left(E_{0}, \nabla_{0}\right)$ to an isomorphism $\psi:(\widetilde{\mathcal{E}}, \widetilde{\nabla}) \rightarrow F^{*}(\mathcal{E}, \nabla)$, both given by gauge transformations in appropriate coordinates of the common base curve $\tilde{X}_{0}$, respectively $\widetilde{\mathcal{X}}$. Therefore we decompose $\psi_{0}$ into an isomorphism $\psi_{0}^{*}:\left(\widetilde{E}_{0}^{*}, \widetilde{\nabla}_{0}^{*}\right) \rightarrow F_{0}^{*}\left(E_{0}^{*}, \nabla_{0}^{*}\right)$ on the punctured base curve $\widetilde{X}_{0}^{*}=\widetilde{X}_{0} \backslash \widetilde{D}_{0}$, and isomorphisms $\psi_{0}^{i}$ defined in neighborhoods of the poles via trivial gluing isomorphisms $\widetilde{\Phi}_{0}^{i}=(\mathrm{id}, I)$ and $\Phi_{0}^{i}=(\mathrm{id}, I)$ respectively :
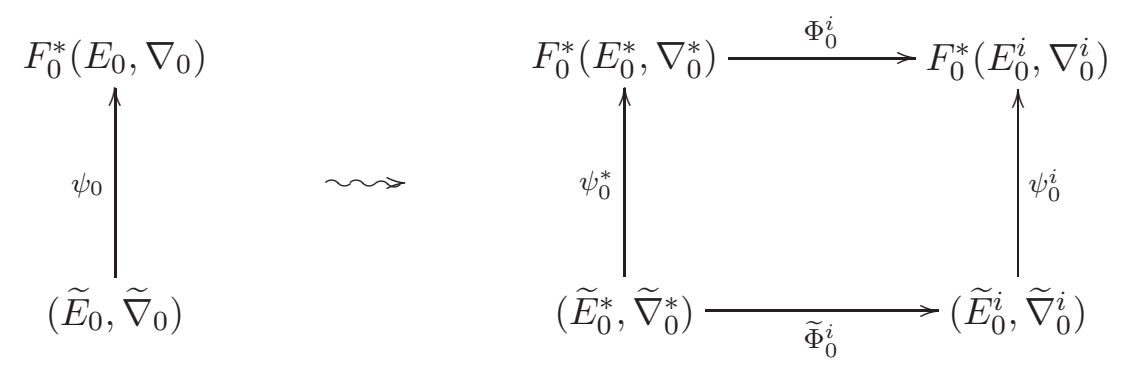

We may decompose $(\widetilde{\mathcal{E}}, \widetilde{\nabla})\left(\operatorname{resp} . F^{*}(\mathcal{E}, \nabla)\right)$ into the connections $\left(\widetilde{\mathcal{E}}^{*}, \widetilde{\nabla}^{*}\right)\left(\operatorname{resp} . F^{*}\left(\mathcal{E}^{*}, \nabla^{*}\right)\right)$ induced on the punctured base curve $\widetilde{\mathcal{X}}^{*}=\widetilde{\mathcal{X}} \backslash \mathcal{D}$, and local connections $\left(\widetilde{\mathcal{E}}^{i}, \widetilde{\nabla}^{i}\right)$ (resp. $\left.F^{*}\left(\mathcal{E}^{i}, \nabla^{i}\right)\right)$ on $\widetilde{\mathcal{U}}^{i}$, together with gluing isomorphisms $\widetilde{\Phi}_{i}=\left(\tilde{\varphi}_{i}, \widetilde{\phi}_{i}\right)\left(\operatorname{resp} . \Phi_{i}=\left(\varphi_{i}, \phi_{i}\right)\right)$. According to lemma 3 we may suppose that in a small neighborhood of the initial parameter in $\mathcal{U}^{i}$ respectively $\widetilde{\mathcal{U}}^{i}$ we have

$$
\left\{\begin{array}{lll}
\left.\left(\tilde{\varphi}_{i}, \widetilde{\phi}_{i}\right)\right|_{\tilde{t}=\tilde{t}_{0}}=(\mathrm{id}, I) & \text { resp. } & \left.\left(\varphi_{i}, \phi_{i}\right)\right|_{t=t_{0}}=(\mathrm{id}, I) \\
\widetilde{\nabla}^{i}=\widetilde{\nabla}_{0}^{i} & \text { resp. } & F^{*}\left(\nabla^{i}\right)=F_{0}^{*} \nabla_{0}^{i},
\end{array}\right.
$$

where $\widetilde{\nabla}_{0}^{i}\left(\right.$ resp. $\left.F_{0}^{*} \nabla_{0}^{i}\right)$ are seen on the vector bundle $\widetilde{E}_{0}^{i} \times \widetilde{T}\left(\operatorname{resp} . F_{0}^{*}\left(E_{0}^{i}\right) \times \widetilde{T}\right)$ over $\widetilde{\mathcal{U}}^{i}$. Moreover, we may suppose by lemma 2 that $\tilde{\varphi}_{i}$ and $\varphi_{i}$ are $\left(n_{i}-1\right)$-jets of diffeomorphisms with respect to the same coordinates on the base curve. In the new coordinates, the connection matrices of $\widetilde{\nabla}^{i}$ and $F^{*}\left(\nabla^{i}\right)$ do not depend on the parameter. Thus $\psi_{0}^{i}$ extends trivially into an isomorphism

$$
\left\{\begin{array}{l}
\psi^{i}:\left(\widetilde{\mathcal{E}}^{i}, \widetilde{\nabla}^{i}\right) \stackrel{\sim}{\longrightarrow} F^{*}\left(\mathcal{E}^{i}, \nabla^{i}\right) \\
\left.\psi^{i}\right|_{\tilde{t}=\tilde{t}_{0}}=\psi_{0}^{i},
\end{array}\right.
$$

given by gauge-coordinate transformations with respect to the coordinate transformation $\varphi_{i} \circ\left(\tilde{\varphi}_{i}\right)^{-1}$. By lemma 4, we know a posteriori that $\tilde{\varphi}_{i}=\varphi_{i}$. In other words, we are still considering a common atlas on the base curve, and $\psi^{i}$ is given by gauge-transformations.

Moreover, $\psi^{i}$ is the unique isomorphism satisfying (9) with respect to our coordinates. Indeed, the first condition implies that such an isomorphism can not depend on the parameter. Thus the second condition provides uniqueness. Note that $\psi^{i}$ is given a priori only in a small neighborhood of the initial parameter. Yet the uniqueness of $\psi^{i}$ implies 
that $\psi^{i}$ can be continued to an isomorphism over $\widetilde{\mathcal{U}}^{i}$ following the analytic continuation of the diffeomorphism on the base curve.

Since $\left(\widetilde{\mathcal{E}}^{*}, \widetilde{\nabla}^{*}\right)$ and $F^{*}\left(\mathcal{E}^{*}, \nabla^{*}\right)$ are two non-singular connections defined on the same base curve and having the same monodromy representation, the Riemann-Hilbert correspondence provides a unique extension $\psi^{*}$ of the isomorphism $\psi_{0}^{*}$, both given by gauge transformations, such that

$$
\left\{\begin{array}{l}
\psi^{*}:\left(\widetilde{\mathcal{E}}^{*}, \widetilde{\nabla}^{*}\right) \stackrel{\sim}{\longrightarrow} F^{*}\left(\mathcal{E}^{*}, \nabla^{*}\right) \\
\left.\psi^{*}\right|_{\tilde{t}=\tilde{t}_{0}}=\psi_{0}^{*} .
\end{array}\right.
$$

We get a commuting diagramm

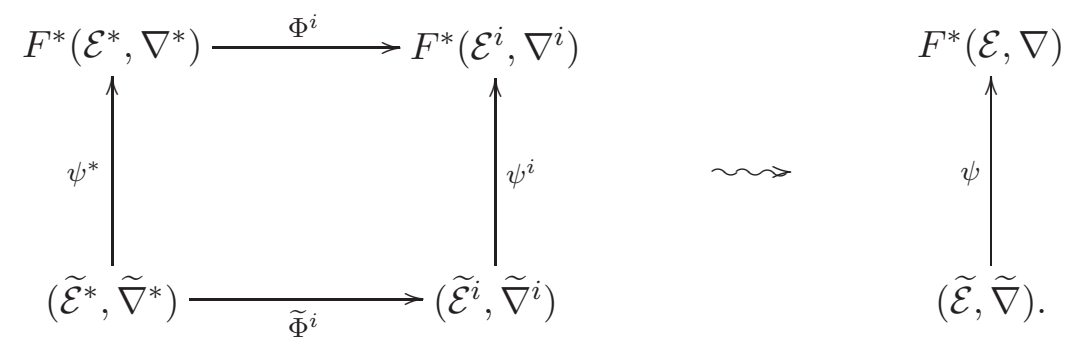

inducing a unique isomorphism $\psi$, given by gauge transformations, such that

$$
\left\{\begin{array}{l}
\psi:(\widetilde{\mathcal{E}}, \widetilde{\nabla}) \stackrel{\sim}{\longrightarrow} F^{*}(\mathcal{E}, \nabla) \\
\left.\psi\right|_{\tilde{t}=\tilde{t}_{0}}=\psi_{0}
\end{array}\right.
$$

\subsection{Unicity}

The argumentation above showed that the triple $(f, F, \Psi)$ is unique if $(h, H)$ is unique. Now the classifying map $h$ is always unique, whereas $H$ is unique if, and only if, there is no non trivial isomorphisms of the universal Teichmüller curve fixing $\left(X_{0}, D_{0}\right)$. This is the case precisely when $(g, m)$ is different from $(0,0),(0,1),(0,2)$ and $(1,0)$. In the cases $(0,0),(0,1),(0,2)$ and $(1,0)$ however, the map $H$ may be composed by untrivial automorphisms of the marked curve. In the case $(0,0)$, the connection $\left(E_{0}, \nabla_{0}\right)$ is the trivial connection on the trivial vector bundle on $\mathbf{P}^{1}$ and any isomonodromic deformation of this connection is trivial. In the cases $(0,1),(0,2)$ with $n \geq 3$, it is possible to define a normalized parameter space $J$ such that there is a unique map $H$ making sure that the image of the map $G$ is contained in this normalized space (see section 4.4). In the case $(1,0)$, it is possible to refer to the case $(1,1)$ and thus restore uniqueness, by fixing a section $\mathcal{T} \rightarrow \mathcal{X}_{\mathcal{T}}$ (see section 4.4).

\subsection{Special cases with automorphisms}

If $3 g-3+m$ is negative, then the dimension of the Teichmüller space is zero. Moreover, in the special case $g=1, n=0$, the dimension of the Teichmüller space is one. The parameter 
space of the isomomonodromic deformation constructed above then is strictly greater than $3 g-3+n$. On the other hand, there are one-parameter families of automorphisms of the punctured curve exactly in these cases. Depending on the context, it may be of interest to take into account those automorphisms.

Assume now $3 g-3+n \geq 0$. In this case, we may restore the universal property of the universal isomonodromic deformation due to the automorphisms.

1 (The case $\mathrm{g}=0$ ). In the case of the Riemann sphere we will be able to diminish the dimension of $T$ by means of a quotient in order to get dimension $\max \{0,3 g-3+n\}$ again. Let us now consider the universal isomonodromic deformation for

$$
m-3<0 \text {, but } n-3>0 \text {. }
$$

Consider a tracefree rank 2 connection $\nabla$ on the Riemann sphere with no poles except 0 and $\infty$, with coordinates $x$ in a neigborhood of 0 and $\tilde{x}$ in a neighborhood of $\infty$, where $\tilde{x}=\frac{1}{x}$.

a) (The case $m=1)$. - Let us consider the case when $\nabla$ has only one pole of multiplicity $n$. We may suppose this pole is $\{x=0\}$. Now apply the construction of the previous section, but in restriction to the following parameter space of local jets fixing zero:

$$
J=\{1\} \times\{0\} \times \mathbf{C}^{n-3} .
$$

The group of automorphisms $\operatorname{Aut}\left(\mathbf{P}^{1}, 0\right)$ of the marked surface $\left(\mathbf{P}^{1}, 0\right)$ acting on our gluing construction is $\left\{\frac{\lambda x}{1-\mu x} \mid \lambda \in \mathbf{C}^{*}, \mu \in \mathbf{C}\right\}$. Such an isomorphism is acting on a jet

$$
x+s_{3} x^{3}+\ldots+s_{n-1} x^{n-1}
$$

in the following way :

$$
\lambda x+\lambda \mu x^{2}+\sum_{i=3}^{n-1}\left(\lambda \mu^{i-1}+\sum_{l=3}^{i} s_{l}\left(\begin{array}{l}
i-1 \\
l-1
\end{array}\right) \lambda^{l} \mu^{i-l}\right) x^{i} .
$$

With help of the automorphisms we may thus recover the whole space of $(n-1)-$ jets from the previous section

$$
\operatorname{Aut}\left(\mathbf{P}^{1}, 0\right) \times J \cong \mathbf{C}^{*} \times \mathbf{C}^{n-2}
$$

and their universal covers will be naturally identified. Yet this new defined isomonodromic deformation will have the universal property.

b) (The case $\mathrm{m}=2$ ). - Let $n_{0}$ (resp. $n_{\infty}$ ) be the multiplicity of the poles of the connection $\nabla$ at zero (resp. at infinity), such that $n=n_{0}+n_{\infty}$. We suppose again $n_{0}>1$. 
In this case, we restrict the universal isomonodromic deformation to the universal cover of the set $J^{0} \times J^{\infty}$ of local jets, where

$$
J^{0}=\{1\} \times \mathbf{C}^{n_{0}-2} \quad J^{\infty}=\mathbf{C}^{*} \times \mathbf{C}^{n_{\infty}-2} .
$$

The group of automorphisms of the marked surface $\mathbf{P}^{1}$ fixing zero and infinity then is $\left\{\lambda x \mid \lambda \in \mathbf{C}^{*}\right\}$. Such an isomorphism is acting on a pair of jets

$$
\left(x+s_{2}^{0} x^{2}+\ldots+s_{n_{0}-1}^{0} x^{n_{0}-1}, s_{1}^{\infty} \tilde{x}+s_{2}^{\infty} \tilde{x}^{2}+\ldots+s_{n_{\infty}-1}^{\infty} \tilde{x}^{n_{\infty}-1}\right)
$$

in the following way :

$$
\left(\lambda x+\lambda^{2} s_{2}^{0} x^{2}+\ldots+\lambda^{n_{0}-1} s_{n_{0}-1}^{0} x^{n_{0}-1}, \frac{s_{1}^{\infty}}{\lambda} \tilde{x}+\frac{s_{2}^{\infty}}{\lambda^{2}} \tilde{x}^{2}+\ldots+\frac{s_{n_{\infty}-1}^{\infty}}{\lambda^{n_{\infty}-1}} \tilde{x}^{n_{\infty}-1}\right)
$$

Again we recover the whole parameter space of the previous section and restore the universal property.

2 (The case $\mathrm{g}=1, \mathrm{~m}=0$ ). Recall that in this case the universal isomonodromic deformation is constructed by suspension. We obtain a non-singular connection on the universal curve having parameter space $\mathbf{H}$. Notice that this connection is invariant under the automorphisms $z \mapsto z+\lambda(\tau)$ of the universal curve

$$
\mathbf{H} \times \mathbf{C} / \sim,
$$

where $(\tau, z) \sim\left(\tau, z+k_{1} \tau+k_{2}\right)$. As a method to restore the universal property, we may fix a supplementary point on the base curve. Let us fix the zero-section $(\tau, 0)$ of the universal curve for instance.

If $3 g-3+n \leq 0$ and $(g, n) \neq(1,0)$, then the initial connection is undeformable, i.e. up to a quotient by the automorphisms we get $\operatorname{dim}(T)=0$ for the parameter space $T$ of the universal isomonodromic deformation. Yet if $3 g-3+n<0$, then there are still automorphisms left, and the classifying map $F$ in the universal property can not be unique.

\section{Complements}

\subsection{Link to the Painlevé equations}

If the initial connection is an irreducible, tracefree rank 2 connection with four non-resonant poles (counted with multiplicity) over $\mathbf{P}^{1}$ and the underlying vector bundle is trivial, then its universal isomonodromic deformation implicitely defines a solution $q(t)$ of the Painlevé equation with the associated initial parameters. Indeed, the global vector bundle $\mathcal{E}$ underlying the universal isomonodromic deformation can be trivialized by bimeromorphic gauge transformations, which are in fact holomorphic in restriction to the parameter space $T \backslash \Theta$ 
(see paragraph 3 in [Mal83b], for example). The exceptional set $\Theta$ is a strict analytic subset corresponding to the set of parameters $t$, such that the associated vector bundle $E_{t}$ is non-trivial. Once the vector bundle is trivialized, one obtains $q(t)$ directly from the system matrix, after a normalization. The parameter space on which the Painlevé equations are defined is the Riemann sphere minus the polar set. Yet the solutions of these equations are well defined only on the universal cover of this parameter space. We remark that our construction provides consistent parameter spaces (see [Oka86]).

\begin{tabular}{|c|c|c|c|}
\hline poles & multiplicity & parameter space & Painlevé equation \\
$x_{1}, \ldots, x_{m}$ & $n_{1}, \ldots, n_{m}$ & $T$ & \\
\hline $0,1, t, \infty$ & $1,1,1,1$ & $\mathbf{P}^{1} \backslash\{0,1, \infty\}=\mathbf{H}$ & $P_{\mathrm{VI}}$ \\
$0,1, \infty$ & $2,1,1$ & $\widetilde{\mathbf{C}}^{*}$ & $P_{\mathrm{V}}$ \\
$0, \infty$ & 3,1 & $\mathbf{C}$ & $P_{\mathrm{IV}}$ \\
$0, \infty$ & 2,2 & $\widetilde{\mathbf{C}^{*}}$ & $P_{\mathrm{III}}$ \\
0 & 4 & $\mathbf{C}$ & $P_{\mathrm{II}}$ \\
\hline
\end{tabular}

However, our construction also applies to the resonant case (of irreducible tracefree rank 2 connections with four poles on $\mathbf{P}^{1}$ ). It would be interesting to see which kind of isomonodromy equations occur in this case.

\subsection{Remarks on connections with trace}

In the spirit of [Mal83b], [Mal83c],[Pal99] and [Kri02], one can also consider isomonodromic deformations of rank 2 connections with varying trace and construct their universal isomonodromic deformations. Consider a meromorphic rank 2 connection $\left(E_{0}, \nabla_{0}\right)$ over a Riemann surface $X_{0}$ with arbitrary trace. On the one hand, the trace of this connection defines a meromorphic rank 1 connection $\left(L_{0}, \zeta_{0}\right)=\left(\operatorname{det}\left(E_{0}\right), \operatorname{tr}\left(\nabla_{0}\right)\right)$ over $X_{0}$. On the other hand, we get a projective connection $\left(P_{0}, \mathcal{F}_{0}\right)=\left(\mathbf{P}\left(E_{0}\right), \mathbf{P}\left(\nabla_{0}\right)\right)$ over $X_{0}$, which is given by a (singular) Riccati foliation on the ruled surface $\mathbf{P}\left(E_{0}\right) \rightarrow X_{0}$ (see [Heu09]).

$$
\left(E_{0}, \nabla_{0}\right) \rightarrow\left\{\begin{array}{l}
\left(L_{0}, \zeta_{0}\right) \\
\left(P_{0}, \mathcal{F}_{0}\right)
\end{array}\right.
$$

Conversely, given a Riccati foliation $(P, \mathcal{F})$, a rank 1 connection $(L, \zeta)$ and a vector bundle $E$ over $X$ such that $\mathbf{P}(E)=F$, we can lift this Riccati foliation to a unique rank two connection $(E, \nabla)$ with trace $(L, \zeta)$. One can see easily that our construction of the universal isomonodromic deformation for the tracefree case generalizes to a construction of the universal isomonodromic deformation for the projective connnection $\left(P_{0}, \mathcal{F}_{0}\right)$. By compactification of the underlying line bundle, the connection $\left(L_{0}, \zeta_{0}\right)$ defines another Riccati foliation on a ruled surface, and we can construct the universal isomonodromic deformation of the trace connection. The universal isomonodromic deformations of $\left(P_{0}, \mathcal{F}_{0}\right)$ and $\left(L_{0}, \zeta_{0}\right)$ then lift uniquely to a universal isomonodromic deformation of $\left(E_{0}, \nabla_{0}\right)$, which is 
defined over a convenient base curve $\mathcal{X} \rightarrow T$. Let us suppose that both $\zeta_{0}$ and $\mathcal{F}_{0}$ have the same polar divisor (counted with multiplicity) as $\nabla_{0}$. Then the parameter space $T$ will have a natural product structure : one factor $\mathcal{T}$ comes from the deformation of the punctured initial curve and will have dimension $3 g-3+m$. The deformation by local jets of $\left(P_{0}, \mathcal{F}_{0}\right)$ provides a second factor $J$ of the parameter space, with dimension $n-m$. As for the deformation by local jets of $\left(L_{0}, \zeta_{0}\right)$, we get a supplementary factor $J^{\prime}$ of the parameter space, with dimension $n-m$. The parameter space $T$ then has dimension $3 g-3+2 n-m$. The so-constructed isomonodromic deformation is the universal object of isomonodromic deformations of $\left(E_{0}, \nabla_{0}\right)$ whose underlying trace-connections and Riccati foliations are locally constant. It is clear that these are isomonodromic deformations in the usual sense. Conversely, for any isomonodromic deformation in the usual sense, the underlying traceconnection and Riccati foliation are locally constant in a neighborhood of generic points of the polar divisor. Here generic points are those where the order of the poles remains constant. The flat connection given by

$$
\mathrm{d} Y=\left(\begin{array}{cc}
\mathrm{d}\left(\frac{t+1}{x}\right) & 0 \\
0 & \mathrm{~d}\left(\frac{t-1}{x}\right)
\end{array}\right) Y
$$

over $(\mathbf{C}, 0)^{2} \ni(t, x)$ is an example of an isomonodromic deformation whose trace is not locally constant at $t=0$. In [Pal99], J. Palmer gave a construction of the universal isomonodromic deformation for the genus 0 case, provided that the leading terms of the connection matrices are non-resonant. As a consequence of their respective universal properties, the construction described above is generically equivalent to Palmer's construction in the rank 2 case.

Acknowledgements. I would like to express my gratitude to my PhD-advisor F. Loray for his constant support, and to thank B. Malgrange and C. Sabbah for their precious suggestions on the manuscript.

\section{References}

[Bri04] Joël Briançon. Extensions de Deligne pour les croisements normaux. In Éléments de la théorie des systèmes différentiels géométriques, volume 8 of Sémin. Congr., pages 149-164. Soc. Math. France, Paris, 2004.

[Del70] Pierre Deligne. Équations différentielles à points singuliers réguliers. Lecture Notes in Mathematics, Vol. 163. Springer-Verlag, Berlin, 1970.

[DZ05] Jean-Paul Dufour and Nguyen Tien Zung. Poisson structures and their normal forms, volume 242 of Progress in Mathematics. Birkhäuser Verlag, Basel, 2005.

[FG65] Wolfgang Fischer and Hans Grauert. Lokal-triviale Familien kompakter komplexer Mannigfaltigkeiten. Nachr. Akad. Wiss. Göttingen Math.-Phys. Kl. II, 1965:89-94, 1965.

[Heu09] Viktoria Heu. Déformations isomonodromiques des connexions de rang 2 sur les courbes. onlinearchiv TEL (http://tel.archives-ouvertes.fr), tel-00358039, 2009. 
[Hub06] John Hamal Hubbard. Teichmüller theory and applications to geometry, topology, and dynamics. Vol. 1. Matrix Editions, Ithaca, NY, 2006.

[Kat76] Nicholas M. Katz. An overview of Deligne's work on Hilbert's twenty-first problem. In Mathematical developments arising from Hilbert problems (Proc. Sympos. Pure Math., Vol. XXVIII, Northern Illinois Univ., De Kalb, Ill., 1974), pages 537-557. Amer. Math. Soc., Providence, R. I., 1976.

[Kri02] I. Krichever. Isomonodromy equations on algebraic curves, canonical transformations and Whitham equations. Mosc. Math. J., 2(4):717-752, 806, 2002.

[LP07] Frank Loray and Jorge Vitório Pereira. Transversely projective foliations on surfaces: existence of minimal form and prescription of monodromy. Internat. J. Math., 18(6):723-747, 2007.

[Mal83a] B. Malgrange. Déformations de systèmes différentiels et microdifférentiels. In Mathematics and physics (Paris, 1979/1982), volume 37 of Progr. Math., pages 353-379. Birkhäuser Boston, Boston, MA, 1983.

[Mal83b] B. Malgrange. Sur les déformations isomonodromiques. I. Singularités régulières. In Mathematics and physics (Paris, 1979/1982), volume 37 of Progr. Math., pages 401-426. Birkhäuser Boston, Boston, MA, 1983.

[Mal83c] B. Malgrange. Sur les déformations isomonodromiques. II. Singularités irrégulières. In Mathematics and physics (Paris, 1979/1982), volume 37 of Progr. Math., pages 427-438. Birkhäuser Boston, Boston, MA, 1983.

[Mal86] Bernard Malgrange. Deformations of differential systems. II. J. Ramanujan Math. Soc., 1(1-2):315, 1986.

[Mal96] Bernard Malgrange. Connexions méromorphes 2 Le réseau canonique. Invent. math., (124):367387, 1996.

[Mal04] Bernard Malgrange. Déformations isomonodromiques, forme de Liouville, fonction $\tau$. Ann. Inst. Fourier (Grenoble), 54(5):1371-1392, xiv, xx, 2004.

[Mat91] J.-F. Mattei. Modules de feuilletages holomorphes singuliers. I. Équisingularité. Invent. Math., 103(2):297-325, 1991.

[MN94] Jean-François Mattei and Marcel Nicolau. Equisingular unfoldings of foliations by curves. Astérisque, (222):6, 285-302, 1994. Complex analytic methods in dynamical systems (Rio de Janeiro, 1992).

[Mos65] Jürgen Moser. On the volume elements on a manifold. Trans. Amer. Math. Soc., 120:286-294, 1965.

[Nag88] Subhashis Nag. The complex analytic theory of Teichmüller spaces. Canadian Mathematical Society Series of Monographs and Advanced Texts. John Wiley \& Sons Inc., New York, 1988. A Wiley-Interscience Publication.

[Oka86] Kazuo Okamoto. Isomonodromic deformation and Painlevé equations, and the Garnier system. J. Fac. Sci. Univ. Tokyo Sect. IA Math., 33(3):575-618, 1986.

[Pal99] John Palmer. Zeros of the Jimbo, Miwa, Ueno tau function. J. Math. Phys., 40(12):6638-6681, 1999.

[Var96] V. S. Varadarajan. Linear meromorphic differential equations: a modern point of view. Bull. Amer. Math. Soc. (N.S.), 33(1):1-42, 1996.

Viktoria Heu

IRMAR

Université de Rennes 1

Rennes, France 